\title{
Using Physical Memorabilia as Opportunities to Move into Collocated Digital Photo Sharing
}

\author{
MICHAEL NUNES ${ }^{1}$, SAUL GREENBERG ${ }^{1}$ AND CARMAN NEUSTAEDTER ${ }^{2}$ \\ ${ }^{1}$ Department of Computer Science, University of Calgary, Calgary, Alberta, CANADA \\ ${ }^{2}$ Computational Science \& Technology Research, Kodak Research Labs, Rochester, NY, USA \\ Email: m.n.nunes@gmail.com, saul.greenberg@ucalgary.ca, carman.neustaedter@kodak.com
}

The uptake of digital photos vs. print photos has altered the practice of photo sharing. Print photos are easy to share within the home, but much harder to share outside of it. The opposite is true of digital photos. People easily share digital photos outside the home, e.g., to family and friends by email gift-giving, and to social networks and the broader public by web publishing. Yet within the home, collocated digital photo sharing is harder, primarily because digital photos are typically stored on personal accounts in desktop computers located in home offices. This leads to several consequences. 1) The invisibility of digital photos implies few opportunities for serendipitous photo sharing. 2) Access control and navigation issues inhibit family members from retrieving photo collections. 3) Photo viewing is compromised as digital photos are displayed on small screens in an uncomfortable viewing setting.

To mitigate some of these difficulties, we explore how physical memorabilia collected by family members can create opportunities that encourage social and collocated digital photo sharing. First, we studied (via contextual interviews with 20 households) how families currently practice photo sharing and how they keep memorabilia. We identified classes of memorabilia that can serve as memory triggers to family events, trips, and times when people took photos. Second, we designed SouvenIRS, a photo-viewing system that exploits memorabilia as a social instrument. Using SOUVENIRS, a family member can meaningfully associate physical memorabilia with particular photo sets. Later, any family member can begin their storytelling with others through the physical memento, and then enrich the story by displaying its associated photos simply by moving the memento close to the home's large-format television screen. Third, we re-examined our design premises by evoking household reactions to an early version of SOUVENIRS. Based on these interviews, we redesigned SOUVENIRS to better reflect the preferences and real practices of photo and memorabilia use in the home.

\section{INTRODUCTION}

Digital photography has become increasingly popular. This is for good reason. It allows numerous photos to be taken and stored, while minimizing the cost and hassle associated with film. People are free to take more photos, increasing their chance of getting a "good" photo, taking playful "candid" shots, and recording event details as memories. They can select and edit their favorites for printing. They are able to store many photos without physical space restrictions. They can easily send photos to others via email or cell phones. Indeed, it is impossible to know just how many photos are taken with digital cameras per year (Norman, 2003) ${ }^{1}$.

While digital photography has revolutionized the way we take photos, we now must consider how such technology affects how people use their photo collections, especially for photo-sharing. As Norman notes:

The technologies of digital picture transmission, printing, file sharing, and display are sufficiently complex and time-consuming as to prevent many people from saving, retrieving, and sharing the pictures they cherish. (Norman, 2003)

Once taken, digital photos are tied to current computing systems that shape and potentially mar our ability to let photos "do what they do". That is, digital photos have altered - and sometimes even lost - many of the affordances that helped create and

1 This paper expands considerably on work first reported at the ACM DIS conference by Nunes, Greenberg and Neustaedter (2008). Additional details of this research are also reported in a thesis (Nunes, 2008). 

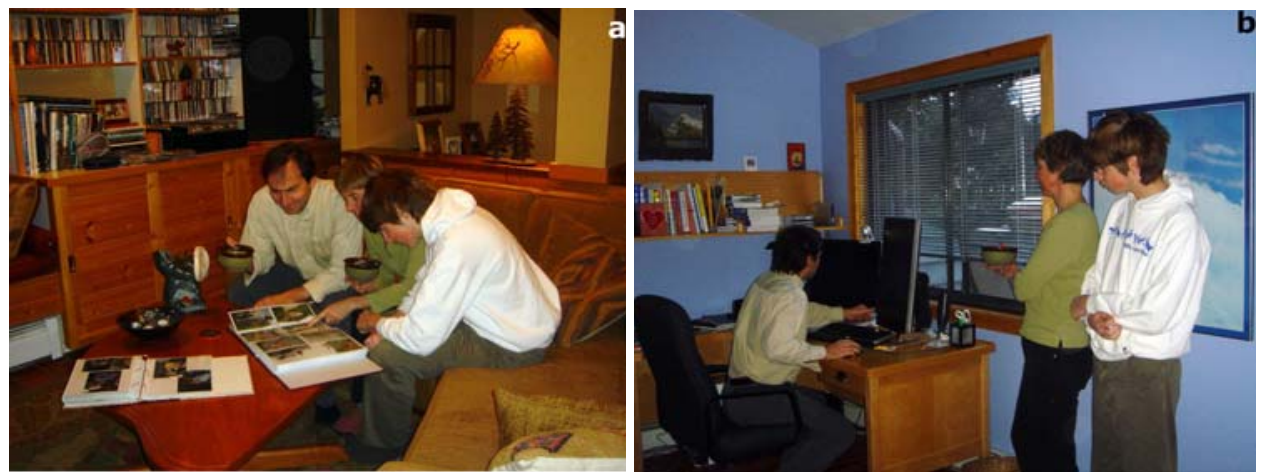

Figure 1: Sharing photos in the home a) with print albums, b) with digital pictures sustain the culture of how we take, use, and in particular how we share print photos (Chalfen, 1987). The challenge for systems designers is to provide digital photos with affordances enabling the best practices that give print photos their value.

Of course, digital photos have their beneficial affordances, especially for encouraging distributed photo sharing (Miller and Edwards, 2007). Tools for sharing photos over the web, via email, instant messengers, social networking and photo sharing sites, combined with the increasingly widespread availability of broadband internet in homes, has made it easier than ever to gift-give photos to distributed friends and relatives, or even various web communities.

Still, many people find that showing print photos face to face in the home is the most enjoyable way to share photos (Frohlich et al., 2002; Lindley and Mark, 2006). In spite of the wealth of photos stored digitally, households rely primarily on printed photo albums for collocated photo sharing (Frohlich et al., 2002). Intuitively, it is easy to see why. Consider the family shown in Figure 1a as an example. This family's print photo albums are located in their living room on a public shelf. Perhaps as part of a conversation, any family member can easily take a photo album off the shelf and onto the living room table. They can easily sit around that album, pointing to photos and discussing them, and pass the album around for a closer look. In contrast, their social use of digital photos is awkward (Figure 1b). They now have to move to their father's home office, as the father (as the primary photo-taker) keeps the family photos in his personal account on the computer located there. This setting is not ideal for family viewing. There is only room for one person to sit in front of the computer desk; the others must stand, sometimes at an awkward angle or distance from the display. Additionally, they must wait while the computer starts, the proper user account is logged into, the desired photos found, etc. The result is that digital photo sharing may be excessively unwieldy, or awkward and not as engaging as print photo sharing. More usually, it just may not happen, as serendipitous opportunities may not present themselves. Our study findings, articulated later in this article, highlight that these and other issues are commonplace for many families.

To recap, the problem is that digital photos are currently difficult to share face to face in the home. As with much of the current research in ubiquitous computing - especially in domestic computing - the challenge is to design computing technologies to fit in with existing routines and practices within the home (Dourish, 2001). The specific question then becomes: how can we design systems that encourage opportunities for face to face sharing in the home that are lost with digital photos?

In this paper, we consider one possible answer: that the tangibility and physical location of home physical memorabilia - souvenirs, keepsakes and mementos - can create opportunities that naturally lead to collocated digital photo sharing. Norman (2003) 
hinted at this potential solution in the juxtaposition of his discussions of souvenirs and photos as memory evoking objects. Of photos he says:

Personal photographs are mementos, reminders, and social instruments, allowing memories to be shared across time, place, and people. (Norman, 2003)

Immediately after, he discusses how souvenirs and mementos are also valued for the memories they evoke:

[A souvenir] is important only as a symbol, as a source of memory, of associations. (Norman, 2003)

Indeed, displays containing both souvenirs and framed photos, such as in Figure 2, are common in many homes (again, articulated in our upcoming findings and also found by Petrelli et al., 2008). This suggests that we might be able to exploit the connection between memory evoking objects by using physical souvenirs as a link to digital photos.

As will be discussed in this paper, we wanted to see how a system designed around such a link could situate and encourage digital photo sharing in the home. Our aim is not necessarily to supplant printed albums; we suspect the practice of printing and organizing subsets of favorites into print albums will remain with some families. Nor is it to suggest that all families keep and use physical memorabilia in a way conducive to photo-sharing. However, given the variety of families and the wealth of digital photos being taken and stored, we hypothesize that our approach is a reasonable way to reintroduce some of the affordances of collocated photo sharing for some homes.

To examine this hypothesis, we conducted contextual interviews with 20 households (Section 2), where we analyze how families currently practice print and digital photo sharing (Section 3), and how they keep physical memorabilia (Section 4). We then describe SOUVENIRS (Section 5), a photo-viewing system that exploits memorabilia as a social instrument. Using SOUVENIRS, a family member can meaningfully associate physical memorabilia with particular photo sets. Later, any family member can begin their storytelling with others through the physical memento, and then enrich the story by immediately displaying its associated photos simply by moving the memento close to the home's large-format television screen. We re-examined our design premises by evoking household reactions to an early version of SouveniRs (Section 6). Based on these interviews, we discuss and critique SouvenIRS and how it can better reflect the real practices of photo and memorabilia use in the home. Related work will be discussed within the context of these sections.

\section{CONTEXTUAL STUDY}

To gain a better understanding of people's existing practices around film and digital photos and to understand how people used and stored memorabilia in the home, we conducted in situ contextual interviews with 20 families. These examined family routines for how they stored and shared photos, and how they used memorabilia.

We state outright that our work was originally design oriented: this study came after we had developed a first version of SOUVENIRS. We were inspired by an idea,

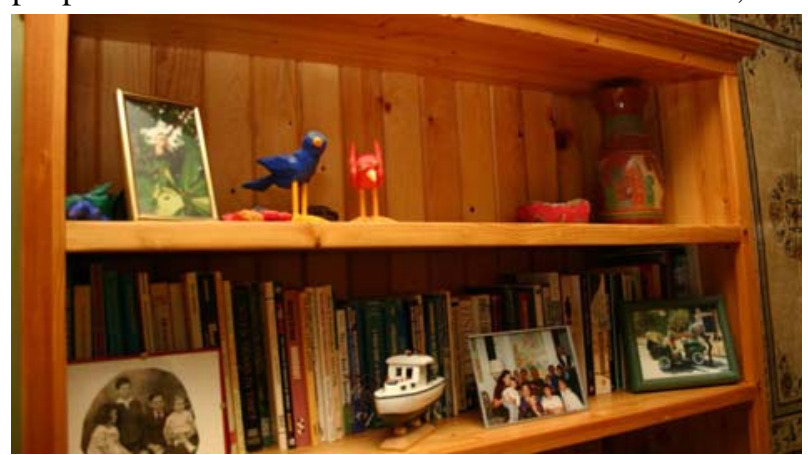

Figure 2: A display of photos and physical mementos 
developed a design rationale, and built SouvENIRS to help us understand the design nuances. As we will see in Section 6, we used this first version as a technology probe (Hutchinson et al., 2003) with our study participants, where we evoked their reactions to a video presentation of this system. We then redesigned parts of SouvenIRS to reflect their reactions as well as what we learnt about household photo sharing and memorabilia practices.

\subsection{Participant Households}

We recruited 20 Canadian households that spanned a range of lifestyles, occupations (e.g., dentists, graduate students, professors, sales engineers, bank managers, etc) and ages (from teenagers to adults in their 50's). The households we selected were all families vs. room-mates or individual occupants. Families were our target audience: they would be more likely to share photographs within the home, and their shared collection of photos would be relevant to all family members (i.e. "family photos"). Yet we were not strict with how we defined our families: our final sample included some families with no children, some families with up to two children, and some families with up to two live-in grandparents. Additionally, all our households had at least one family member who took and stored digital photos on a regular basis. We tried to schedule families so that all family members would be available to participate. While not possible in some cases, most of our sessions did include all family members.

\subsection{Method}

We used semi-structured contextual interviews (Beyer and Holtzblatt, 1998), each approximately one hour long. Children under 14 years were not interviewed due to ethics concerns. Interviews were held in participants' homes, as this would allow them to recall and describe their routines for photograph and memorabilia storage and sharing in context. It also allowed us to gain a first-hand view of how these practices fit in the domestic environment.

We first asked household members how non-digital and digital photos were organized, displayed, and shared within and outside the home. Next, we asked household members what types of memorabilia - souvenirs, keepsakes and mementos - they collected, what memories were associated with these, and how they displayed them in the home. To ground our interviews within actual practices, we asked participants if they would show us (and if we could photograph) the location and the types of photographs and memorabilia they kept (Figure 2 is an example). Interview and touring sessions were semi-structured in order to be opportunistic. We often used our questions to probe participant's actual context or asked if we could be shown a particular collection or display as it came up in the interview. We also asked participants about collections or displays as we came across them in the tour. Finally, we showed families a video demonstration of SouveniRs. They told us what they thought of it, how they might see themselves using it, and what they disliked or might want to see changed or added to it. This aspect of the study is discussed in Section 6.

There were several benefits to conducting the interview sessions within the context of participant homes. We were able to gain a first-hand view of where participant collections were stored and displayed within their homes without relying on participant descriptions of these areas. Also, in being shown the collections we became involved as observers in a sharing event, and, while these were artificially caused, it is likely that real sharing events would involve similar actions. Additionally, participants could discuss their collections in place rather than having to recall descriptions from memory. 


\subsection{Data Collection and Analysis}

All interviews were audio recorded and handwritten notes were taken to aid analysis. We used the well-known open coding qualitative method (Strauss and Corbin, 1998) to analyze this data, and generated a large amount of codes that reflected a variety of household routines. We then used affinity diagramming (Holtzblatt et al., 2005) to categorize these codes and pull out important themes. These themes, rather than the individual codes, form the heart of our result section. However, we do provide an overview of our codes in subsequent sections to help ground future research in this space. Our findings are presented in three parts: use of digital vs. print photography, use of memorabilia, and reaction to our system design.

\section{PHOTO SHARING IN THE HOME}

The first part of our study was aimed at gaining a better understanding of people's existing practices around film and digital photos. We briefly present the related literature surrounding photo routines and then articulate our own interview findings.

\subsection{Related Literature}

Our research targets a specific culture around photography known as the Kodak culture (Chalfen, 1987). Here, photography is undertaken by ordinary people (as opposed to professional and hobbyist photographers) who use photographs to participate in home mode communication: "a pattern of interpersonal and small group communication centered around the home." This home mode emphasizes storytelling as a dominant feature of how photos are interpreted and shared (Chalfen, 1987).

While the Kodak culture is adopting digital photos, this does not imply that the Kodak culture embraces all digital photo methods. For example, Miller and Edwards (2007 found that websites for digital photo sharing, such as Flickr.com, are largely unadopted by the Kodak culture. Indeed, another culture (which they call Snaprs) use these sites primarily for showing off photographs (rather than family snapshots) to strangers. In contrast, Kodak culture people often relied on printed versions for sharing, but showed a preference for using e-mail (instead of web sites) when sharing digital photos. This was because they prefer private photo sharing with family and friends, as opposed to the Snapr notion of online public photo sharing. This suggests that Kodak culture families would highly value technologies that can improve the manner in which they are able to share digital photos in their homes with family and friends, vs. strangers.

Other researchers have examined co-present storytelling over photos to elicit requirements for photoware: software aimed at photo sharing and organization. Frohlich et al. (2002) found that co-present sharing is an enjoyable activity because it allows people to relive experiences, share them with others, and jointly reminisce. While performing these acts, people also had a strong preference for sharing prints because of their manipulability. Crabtree et al. (2004) investigated co-present storytelling further and articulated the importance of being able to gesture at photos and create situated arrangements (e.g., various piles of photos that designate public or private viewing). Building on this, Lindley and Mark (2006) found that prints were preferred for facilitating these types of interactions. They also found that people like viewing photos on large, high-resolution displays in social spaces (e.g., the living room), but dislike the need for crowding around a laptop or monitor when showing to larger groups. Slide show modes for showing digital photos were also seen to inhibit conversation by restricting the flow of sharing (Lindley and Mark, 2006).

While our research looks to encourage co-present sharing in the home, archiving and organization practices are worth considering as they affect how photos are made available for sharing. Frohlich et al. (2002) found that archiving prints mainly involves the culling 
and placement of photos into albums. However, in actual practice, this task becomes tedious and time-consuming, and, in many cases, is left undone. For digital photos, Rodden and Wood (2003) and Kirk et al. (2006) found a reliance on simple date/event based folder schemes. This works because people typically browse recent $v s$. old photos, so searching for a particular photo does not involve looking through the entire collection. Folders can also be used to narrow a search to a particular time or event. In this regard, Bentley et al. (2006) noticed that users will sometimes stop photo searching when a "good enough" photo is found, rather than continuing to search for a more optimal photo. They also noticed the act of sidetracking: photos encountered during searching can change the direction of the search, providing more serendipitous opportunities for photo browsing. These results suggest that query searches for specific photos may not be necessary for personal/family photo collections.

Photos displays in the home can be considered as a means for both archiving and copresent sharing. Kim and Zimmerman (2006) found that two types of displays were used in homes: formal, and informal. Formal displays refer to professional posed photos (e.g. graduation photos, family portraits, etc.) placed on display. While these offer potential to start conversation with guests, it is the personal and candid informal displays that provide greater opportunity for storytelling. Swan and Taylor (2008) looked at particular examples of photo displays, and how their arrangements and properties convey meaning to home inhabitants and guests. For example, the positioning of two framed photos - one prominently visible, one somewhat obscured - can relay a message of the relative importance placed on them. The message may or may not have been intentionally created, but, nevertheless, artifacts of print photo displays in the home can convey impressions of the home and affect the storytelling narratives given around the displays (Taylor et al., 2006).

\subsection{Overview of Analysis Codes}

Our interviews about print and digital photos build on the related work by focusing on how families manage, store, and share their photo collections. In particular, we were interested in how various technologies and techniques for photo management created opportunities and influenced families' abilities to share their photos. The result of these interviews was a large body of discussions about families' practices and experiences with their print and digital photo collections.

We used open coding to analyze and pull themes from these discussions - an overview of the codes is shown in Table 1. A full listing of the codes is available in Nunes' thesis (2008); the table is a summary that breaks the analysis into five main themes, giving subcategories for each theme along with example codes. The table highlights some of the ways we saw families describing their practices with photos, and how we were able to break these descriptions down for use in our analysis. While our results will be presented in later sections, this piece of our analysis is relevant to consider for future research into domestic photo routines (e.g., as a basis for focusing inquiry or analysis). The five main categories shown in the table are briefly described below.

- Photo types (Table 1a) describe the kinds of photos comprise a particular collection. Participants tend to take particular types of photos, and the type influences what they choose to display or share with others. Participant's descriptions of types often included the occasions that prompted photo taking, the content of the photos themselves, or meta-information about photo sharing, (e.g., giving duplicates to a person in the photo).

- Storage locations (Table 1b) describe the physical locations where collections are kept. Descriptions typically include high-level areas in the home (i.e., rooms), or 
lower level information about particular containers in which photos are kept (e.g. shelf, drawer).

- Storage types and strategies (Table 1c) describe how photo collections have been organized and managed. This typically includes information about the physical form of storage, organization schemes, and rationales for the photo storage routine.

- Likes/dislikes/challenges (Table 1d) refer to descriptions of participants' experiences in managing and sharing their photo collections. This includes issues that affect the potential for sharing photo collections, issues relating to how easily photo collections can be organized, kept safe, and easily accessed. This also includes issues in dealing with digital technology needed for managing digital photos, and experiences with the tangible form of prints.

- Finding strategies (Table 1f) refer to participants' descriptions of how they would go about finding particular photos as a precursor to sharing. This typically includes using explicit markings (such as when an album has been labeled), implicit markings (such as knowing that a photo set is in a particular style of album), or having to browse photos in order to find a desired set.

\subsection{Organization and Location}

We now describe the practices we saw surrounding these coding categories. For our purposes, we distinguish photographs in terms of how they are stored vs. how they are taken. During our interviews, we found that people discussed photos based on how they are stored and used, rather than on what camera (digital or film) they used to take the photograph. In particular, families who maintained print photo albums after switching to a digital camera treated them as print photos, and often had only a fuzzy idea of which prints were originally film or digital. Similarly, those who scanned in old prints as digital copies treated them as digital photos. To understand the differences in use between these two types of media, our results focus on comparing the routines surrounding print photos with digital ones.

Much of what we found replicates and confirms previous findings. For brevity, we restrict our presentation to those aspects that go beyond what was previously shown, or that emphasize prior findings related to issues in photo sharing.

Print photo collections were typically stored in photo albums, photo envelopes, and photo boxes (Frohlich et al., 2002). We found that photos in these collections were usually organized chronologically, or by event, or some combination of both; however, the amount of explicit 'organization' varied considerably. Time often served as the primary organizational means, as photos are, by default, organized and grouped chronologically when they are developed (on a film roll basis).

Within these container objects (e.g., albums, envelopes), print collections were stored in various places around the house, the most common being an office or living room shelf. They also showed up in bedrooms or basements, in closets or cabinets or other places. When asked why the photo collections were located where they were, participants mainly cited that their choices were usual a function of finding adequate space and avoiding clutter. Some, however, did note that locations were chosen to be easy to get at when guests were around.

[Because] it's a shelf (laughs). That's probably the most used room and when people come over you're usually sitting in the living area somewhere...it's easy and accessible to go and grab. - P16, Wife 
Table 1: Sample analysis codes for print and digital photo collections.

\begin{tabular}{|c|c|c|}
\hline a) Photo Types & Example Codes & Description \\
\hline \multirow[t]{3}{*}{ Occasions for photo-taking } & [Trips] & $\begin{array}{l}\text { Vacations or other events that involve } \\
\text { going places }\end{array}$ \\
\hline & [Special Events] & Parties, ceremonies, performances, etc. \\
\hline & [Day-to-day] & $\begin{array}{l}\text { Around the home, candid, day-to-day } \\
\text { pictures }\end{array}$ \\
\hline \multirow[t]{3}{*}{ Content of the photos } & [Friends] & Photos of friends \\
\hline & [Architecture] & Buildings \\
\hline & [Scenery] & Photos of nature, etc. \\
\hline \multirow{3}{*}{$\begin{array}{l}\text { Meta-info about particular photos } \\
\text { being stored or shared }\end{array}$} & [Copies] & Doubles of prints \\
\hline & [Relevant] & $\begin{array}{l}\text { Photos relevant or interesting to the } \\
\text { people they are being shared with (e.g., } \\
\text { shared experience, common interest) }\end{array}$ \\
\hline & [Recent Photos] & Photos taken recently \\
\hline b) Storage Locations & Example Codes & Description \\
\hline \multirow{3}{*}{ High-level area (i.e. room) } & [Office] & A home office \\
\hline & [Living Room] & The family living area \\
\hline & [Parent House] & Items stored in a parent's house \\
\hline \multirow[t]{3}{*}{ Low-level container } & [Shelf] & Placed on a shelf \\
\hline & [Drawer] & Placed on a drawer \\
\hline & [Fire Safe] & Kept in a fire-proof safe \\
\hline \multirow{4}{*}{$\begin{array}{l}\text { c) Storage Types/Strategies } \\
\text { Physical form of storage }\end{array}$} & Example Codes & Description \\
\hline & [Album] & Kept in albums \\
\hline & [Loose] & Loose photos \\
\hline & [DVD] & Stored on a writable DVD disc \\
\hline \multirow[t]{2}{*}{ Organization Schemes } & [Event] & Stored by event \\
\hline & [Chronological] & Stored in order by date taken \\
\hline \multirow[t]{3}{*}{ Rationales for storage choice } & [Available Space] & $\begin{array}{l}\text { Location chosen because it provides } \\
\text { adequate spaces to store the items }\end{array}$ \\
\hline & [Near Guests] & $\begin{array}{l}\text { Stored in a place near where guests } \\
\text { would be }\end{array}$ \\
\hline & [Pragmatic] & $\begin{array}{l}\text { Pragmatic reasons (e.g. because it's a } \\
\text { shelf, or photos hung where there are } \\
\text { studs in the wall) }\end{array}$ \\
\hline d) Likes/Dislikes/Challenges & Example Codes & Description \\
\hline \multirow[t]{2}{*}{ Potential for sharing } & [Send Link] & $\begin{array}{l}\text { Sending a link, rather than an email } \\
\text { attachment }\end{array}$ \\
\hline & [Screen Viewing] & $\begin{array}{l}\text { Viewing on screen presents difficulties } \\
\text { (e.g. viewing angle, brightness, etc.) }\end{array}$ \\
\hline \multirow[t]{2}{*}{ Organization } & [Avoids Clutter] & Storage mechanism avoids clutter \\
\hline & [Hard to Caption] & $\begin{array}{l}\text { Hard to write captions or labels for digital } \\
\text { photos }\end{array}$ \\
\hline \multirow[t]{2}{*}{ Safekeeping } & [Prevent Damage] & $\begin{array}{l}\text { Storage mechanism helps to prevent } \\
\text { damage to the photos }\end{array}$ \\
\hline & [Archival] & $\begin{array}{l}\text { Will the format be archival? Will the } \\
\text { photos still be viewable in } 50 \text { years? }\end{array}$ \\
\hline Accessibility & [Easy Access] & Easy to get at the photos \\
\hline \multirow[t]{3}{*}{ Technological issues (digital) } & [Loading Time] & Time taken to load up digital pictures \\
\hline & [Slow Connection] & $\begin{array}{l}\text { People with slow internet connections will } \\
\text { have a hard time sharing photos }\end{array}$ \\
\hline & [Unreliable] & $\begin{array}{l}\text { Loss of access due to system failure (e.g. } \\
\text { online site is down, or system crash) }\end{array}$ \\
\hline \multirow[t]{2}{*}{ Tangible Form (prints) } & [Hides Technology] & $\begin{array}{l}\text { Technology behind photo showing is } \\
\text { 'hidden' when sharing prints }\end{array}$ \\
\hline & [Fun To Search] & $\begin{array}{l}\text { Searching through photos is enjoyable, } \\
\text { get to look at the others you might not } \\
\text { have been looking for }\end{array}$ \\
\hline e) Finding Strategies & Example Codes & Description \\
\hline \multirow{2}{*}{ Explicit Markings } & [Date] & Might find searching by date \\
\hline & [Album Label] & Might be able to find in a labeled album \\
\hline Implicit Markings & [Album Style] & $\begin{array}{l}\text { Style of the album (i.e. cover color), can } \\
\text { remember contents from appearance }\end{array}$ \\
\hline \multirow[t]{2}{*}{ Browse } & [Guess] & $\begin{array}{l}\text { Would have to guess where a picture } \\
\text { might be }\end{array}$ \\
\hline & [Flip Through] & Would have to flip through photos \\
\hline
\end{tabular}


Sometimes, photos were placed in a temporary location. This included the storage of photo envelopes waiting to be organized into albums, or photo albums that had been recently taken out to be shared with guests and not yet returned to their normal location.

Location also includes how and where photos are placed on display within the home. As expected, most framed photos are placed on walls or shelves. Less formal unframed photo displays (loose photos) might be placed on a fridge door (Kim and Zimmerman, 2006). We most often saw photo displays in living rooms, but they were also common in hallways, bedrooms, dining rooms, kitchens and staircases (Kim and Zimmerman, 2006). These locations served the purpose of providing serendipitous opportunities for photo sharing as photos may be noticed by guests if they are placed on display in a common area of the home.

We asked participants why display locations were chosen. Their reasons primarily concerned how the display fit in, where there was available space to display the photos in question, as well as pragmatic reasons such as the existence of a shelf or support studs in the wall.

I thought this was a good spot. It's just for us, it's not for showing off, you know.

I just thought this was a good spot for the size of the picture" - P1, Mother

Digital photo collections, unsurprisingly, were mainly kept in folders on a computer. All but one family simply used the file system rather than special purpose software for organizing their photos, saying this was adequate. Some participants also kept photo copies on an online site as it allowed for easier sharing with remote family/friends. Others regularly stored photos onto CD or DVDs (Frohlich et al., 2002).

To summarize, in contrast to print photos, the location and storage of digital photos is problematic for photo-sharing. Because digital photos are strongly tied to the computer they are kept on and, thus, its location, they are not as easily accessible for sharing. It is only with a considerable effort that this might be overcome, e.g., photos could be transferred to a laptop to be shown, or written to a DVD to be shown on a home television. One participant notes how this effort inhibits opportunities for sharing photos at a birthday party:

You've got to go to the computer right, you can't just go to the kitchen table with it. Which is where - we had my birthday party, remember, where'd everybody hang out? We've got all that space and all that space and everybody was just right here. Unless you have a laptop with wireless, which we don't have..." P16, Husband

\subsection{Family Member Roles}

Next, we wanted to determine the roles of family members when storing and organizing the photos. Specifically, we wanted to see: if there was a primary photo organizer; if knowledge of photo collections was largely relegated to that organizer; and whether other family members shared knowledge of these collections, which in turn would allow a particular member to recall, find and show photos stored and organized by someone else. We expected significant differences between print and digital photo collections.

In print photo collections, the roles varied by family type. For families with children, we found that photo organization, such as photo albums, was primarily undertaken by a single parent - usually the mother. Although others would sometimes help in the organization, this was usually in a secondary role. What was striking was that in most instances all household members were aware of the collections, even though the collection was maintained by a single person or family subset. This was typically because the collections were stored in common areas of the home shared by all members of the 
household (e.g., living room, shared home office), thereby creating and promoting shared household knowledge.

Households consisting of younger couples differed. This was generally because the couple had not been together as long, and the idea of a shared family collection had not yet evolved and solidified. These participants often had personal photo collections that had been kept and maintained separately, where the collections began prior to couplehood. Thus, the collections were largely personal and perhaps less relevant to the other person. As a result, shared knowledge of these collections varied. In instances where personal albums had been placed on shelves throughout the home, we saw that participants did share knowledge of them.

Digital photo collections containing family pictures were also maintained by a single parent in a family with children, but this time more often by the father. In sharp contrast to print photos, knowledge of collections was generally limited to the person who had organized them. While some family members may know that a photo collection was being kept, they often did not know what was there or how to access them. Because our interview protocol asked a family member to show us the digital photo collections in the presence of other family members, this lack of knowledge was often revealed as in the quote below:

Father: [after showing the mother some folders containing photos] Did you

know that?

Mother: No, I don't use that, I don't know. - P1, Mother and Father

Even when participants knew a photo collection was being kept, there was reluctance expressed about accessing a collection maintained by another family member. This reluctance was because photos were managed under individual user accounts, or even computers, which were seen as private - belonging only to the owner.

I'd use [my husbands] computer but it's his computer. I know his password but it's like his space. And my computer is my space - P4, Wife

Because access to the digital photo collections was often limited, we saw that the primary organizer usually took on the role of a librarian for the family photos. If another family member wanted access to a particular photo or set, they would resort to a social channel for access, relying on the primary organizer to retrieve the photos for them.

I don't think they even know about the organization. Usually when they wanted some [photos] - like for her project, she'd ask 'Mom, can I have', you know, a picture of her in an occasion. And then I will find it and I will get her a copy P8, Mom

Only one of our families maintained photos collectively, where they chose to store them in the common root folder C:/photos. Most other families used a folder held within a user account, typically My Documents/My Pictures. However, older children (e.g., teens) who took photos tended to maintain and control their own digital photo collections quite separately from the family collection.

To summarize, both print and digital photos had a primary organizer. With print photos, photo-sharing is easy as all were aware of the collection and how to access it. With digital photos, photo-sharing proved difficult, as family members often did not know about the collection or how to access it, or were reluctant to access them because they were stored in personal accounts. 


\subsection{Why and How People Share Photos}

When we looked at how families chose photos to share (Chalfen, 1987), we noticed a correlation between their motivation for photo sharing and the means they used.

1. Displayed photos are those placed out in the open, typically in frames hung on walls or placed on shelves. These photos are readily noticeable by anyone within their general vicinity. In this way can be shared implicitly.

2. Shown photos are those that an owner brings out specifically to share with others, e.g., a print album of vacation pictures taken from a shelf and shown to guests, or a wedding slideshow (slides or digital) shown to relatives.

3. Gift-giving photos are given as gifts to the recipient. Gift giving does not necessarily involve face to face sharing; recipients are free to look at the photos at their convenience. Examples include mailing or handing off printed doubles, or emailing digital photos to friends.

For displayed photos, family members typically chose photos based on aesthetic vs. communicative reasons. That is, they chose photos that were personal favorites, where the idea that guests would also see them was not the main influencing factor.

Everyone has their favorite ones that they want to print and display. In fact I have a bag of negatives because I think - this is just a rental property - but I think I'm gonna have a house someday, and I'm gonna want to blow this one up and frame it. - P17, Wife

For shown photos, we asked family members about why they showed particular photos to guests (Chalfen, 1987). Their reasoning typically included the guest being in the pictures, or the guest having shared the experience or event shown in the photos, or that it led naturally from social relevance (e.g., conversations about particular vacation destinations). Thus the particular guest and the course of conversation are the primary factors, rather than simply showing some recent liked photos.

Just if we thought it would be interesting to someone else. Like if there are people we did sports with or hiked with - like our mountain trips. Or if we're traveling somewhere - someone that's interested in hearing about the trip, we'd show.- P5, Father

For gift-giving, participants often stated that photos were chosen and given as a "status update" to infrequently seen family and friends, i.e., to provide interpersonal awareness to intimates living outside the home (Neustaedter et al., 2006). Photos typically illustrated recent life events, or present new photos of children growing up.

My parents...anytime they take pictures they make us copies ... and we get copies of our nieces and nephews or our kids that they've taken. Usually it's kid related. - P5, Mother

I was living in a resort at the time, so I was sending them to my family and sharing them with them that way...I would send the scenic pictures because, of course, the mountains are so beautiful. And I would also send them, like if I had a friend I talked about a lot, I would send 'oh, this is...we work together'. - P17, Wife

Print vs. digital photo-sharing. The differences between print and digital photos affect how people share photos. A summary of our findings for the methods and motivations for sharing photos and how they are amenable to print and digital technologies is given in Table 2. At one extreme, physical prints are currently more amenable as displayed photos (Table 2, Row 1). While digital photo frames are readily available, they are still 
Table 2: Photo sharing methods and their amenability to technology.

\begin{tabular}{|l|l|l|} 
Photo Sharing Method & Most Common Motivation & Most Amenable Technology \\
\hline Displayed Photos & Aesthetics/Favorites & Print \\
\hline Shown Photos & Social Relevance & Print and Digital \\
\hline Gift-giving & Interpersonal Awareness & Digital \\
\hline
\end{tabular}

expensive and constrained by power, lighting conditions, and limitations on how they can be positioned in a particular space. Indeed, there is some evidence suggesting that digital photo frames are ill-conceived as replacements for print photos (Swan and Taylor, 2008). Only one of our families owned a digital photo frame, and they stated that this was kept off most of the time:

Well we used it at Christmas when we first got it. And we actually think we'll take it with us the next time we travel - cause you can see the pictures a little better on that rather than on the digital camera...I think we probably would use it more if we were having a bunch of people over. But when it's just us, we don't bother plugging it in. - P9, Wife

Two participants noted that they used their computer's desktop wallpaper or screensaver to display a digital photo, but of course its placement was restricted to that computer's location. At the other extreme, digital photos are seen as much more effective for gift-giving (Table 2, Row 3). Compared to the cost and effort of print duplication, it is very easy to email others photos and links to online albums. Shown photos are still amenable to both print and digital media (Table 2, Row 2); while many participants liked showing slideshows on computers or laptops, those who maintained print photo albums preferred to show those.

As mentioned earlier, new cultures have emerged around sharing photos on web sites such as Flickr (Miller and Edwards, 2007). In form, this kind of sharing resembles giftgiving, however it seems to be centered on sharing aesthetically-pleasing photos, although often with "strangers."

Sharing and Tangibility. Notwithstanding the above, we were somewhat surprised by how many people still chose to print photos that they had taken with a digital camera. For most, the affordances of a paper print, especially for showing, was superior and still had great appeal. First, printed photos offered easy viewing. Participants reported that printed photos hide the technology that would otherwise be visible when viewing on a computer. This could include the physical appearance of the computer, the necessity of navigating a GUI in order to find and display photos, or the lower image quality when viewing on a computer monitor relative to a print. People also said they preferred not to use computer technology when showing photos to older relatives, as they may not be comfortable with that technology.

Efforts in finding and navigating to digital photos on the computer, invoking the correct application to view them, or selecting and placing photos on another media to be shared are avoided. By way of contrast, consider the example below, where an envelope of print photos has to be retrieved and brought to the person to be shown. However, there is no further preparation involved; the prints are always ready to be shared.

If you want to show [digital photos] to somebody it's harder, cause you have to bring them to your computer or burn a CD and take it to them. It's a bit more effort than just [print photo] envelopes. - P4, Wife

The tangible form of the photo album was also reported to have a positive effect on the social engagement of families and friends when sharing photos within the home. This tangible engagement is revealed in the two quotes below. 
I really like having them there to look at... just having it more like a book so you can socially sit and go through things with like my mom and friends - P5, Mother.

I really like to be able to grab something and hold on to it and look at it and pass it around. Where, the digital, you stick it all on a CD and it takes less space, but you don't do anything with them. - P16, Wife

A physical photo album can be held and passed around, and this ability actively engages those who are being shown the photos. Showing the photos on the computer may resemble more of a presenter and audience relationship, as those being shown the photos have no control and are less likely to become involved. Another social benefit of prints, reported by other researchers (Crabtree et al., 2004, Lindley and Monk, 2006), is that the arrangement when showing photos on a desktop computer prevents the presenter from seeing the reactions of those being shown the photos.

\subsection{Discussion}

In summary we can see that digital photos have significant problems when it comes to co-located photo sharing, all of which stem from their abstract and hidden nature and lack of true physicality. Print photos, on the other hand, fit co-located sharing well because of their physicality.

1. Location: Print photos are typically easy to access because they are in printed form and placed in areas that frequent sharing activities. This makes them visible and ready at hand for sharing. Digital photos on the other hand are located on computers that are hidden, hard to access, and not necessarily in locations amenable to sharing.

2. Serendipity: Print photos are easily placed on display in homes and this creates opportunities for serendipitous conversation and photo discovery. In contrast, digital photos are not readily visible in the home to spark such spontaneous sharing opportunities. Even if a computer is located in common room of the home (e.g., a kitchen), people have to setup specialized screensavers/software to show photos and this is only visible if the computer is not in use.

3. Accessibility: Both print and digital photos are typically maintained by one family member; however, the placement of print photos in a public home location means that other family members know about the photos and can access them. In the case of digital photos, knowledge of them by family members other than the primary organizer is limited and people are reluctant to search for them. Computer access restrictions further circumvent other family members from finding digital photos. One can certainly print digital pictures, yet doing so for all of one's photos is not a reasonable option for most. It is expensive, and it does not scale well to the myriads of photos that people now take.

4. Ease of Viewing: Print photos can be quickly shown to others and people actually prefer to touch and pass around tangible representations of their memories. This certainly contrasts with digital photos, which do not typically have any physical form factor. Of course, digital photos could be presented on a laptop or tablet PC that is passed around, but this does not get around navigation issues involving computer software. That is, to display any photo, users must first navigate through various windows, menus, or dialogs. This is somewhat akin to flipping pages in a photo album to find a desirable photo for sharing, yet the fact that one is viewing photos as they browse (found to be desirable) is much different than viewing applications and windows unrelated to the act of photo browsing. 
We also saw that people share photos for different reasons and using different means. In the digital realm, gift giving is already well handled by asynchronous messaging systems (email, instant messengers) and by special purpose web sites. Displayed photos are now being addressed by digital photo frames, although its general acceptability still has a long way to go. Shown photos are the sweet spot for any system that links physical memorabilia with photo collections. As we will show later, this is where memorabilia could play a role in promoting easy sharing of digital photos in a socially engaging location.

\section{PHYSICAL MEMORABILIA: SOUVENIRS, MEMENTOS AND KEEPSAKES}

The next stage of the study investigated participant families' practices with physical memorabilia. Again, we first present the related literature and then articulate our own interview findings.

\subsection{Related Literature}

Souvenirs, mementos and keepsakes are all types of physical memorabilia, i.e., an object deliberately kept by a person as a reminder of a person, place or event, and which are directly meaningful to their memories (Petrelli, Whittaker and Brockmeier 2008). We use these terms somewhat synonymously in our discussion below.

Several researchers have studied the collection, use, and importance of memorabilia. Matching the definition above, Csikszentmihalyi and Rochberg-Halton (1981) found that souvenirs, heirlooms, and mementos were amongst some of the most cherished objects in the home because of their associated memories. Yet their work did not provide a detailed discussion of these objects; instead it looked at the broader meanings and sense of self created by objects in the home. A focus group conducted by van den Hoven and Eggen (2005) to define "souvenir" turned up a variety of potential meanings: objects symbolizing relationships between people, places, moments, etc.; objects that have emotional value; or, objects used to evoke memories. What is notable about all three of these definitions is that each involves "physical objects to which memories are attached." The focus group also revealed that participants' most valued souvenirs were often kept on display in the living room. Of 30 participants, 22 reported having media related to their most valued souvenirs: photos were most common, but other music and video were also reported.

The work of Petrelli, Whittaker and Brockmeier (2008) is highly related to our own study. Both were conducted independently but in parallel, and used very similar methods (although their subject pool was British vs. Canadian). Both were reported at conferences in within a few months of each other (our initial report in Nunes, Greenberg and Neustaedter 2008 precedes theirs by a few months). Both have overlapping results, but each contributes richness not found in the other. In particular, their work considers how digital technologies for evoking memories can be designed by leveraging the role that physical objects already play as mementos in the home. They too suggest - albeit much more generally - that digital technologies could be integrated with memorabilia to better support sharing memories with others, integrating memories within everyday life, and revealing personal memories for reflection. They describe the arrangement of these objects within the home as constituting an autotopography, i.e., "a physical map of memory, history and belief'. It is beyond the scope of this paper to summarize all their findings, but we strongly recommend that it be read as a companion paper that complements the work reported in this section. Suffice to say, their overall findings also re-enforce the rationale for a system such as the one we will describe in Section 5. 


\subsection{Overview of Analysis Codes}

Our own investigation of memorabilia centered on three key points.

1. Types of Memorabilia: What items are kept and collected? What size are they? How easily can they be moved?

2. Location: Where were items located throughout the home? How easily could they be accessed? How noticeable are they to guests?

3. Memory Association: What memories are associated with items? Are memories associated with both the items as well as particular photo sets? How easily could the physical items be linked with photo collections?

We used the same coding method as we did for coding family photo routines - an overview of codes is shown in Table 3. Again, we do not list all codes (see Nunes 2008 for that) and instead highlight several that could act as a basis for future investigations. The five most prominent themes from our data are shown in the table are briefly described below.

- Memorabilia types (Table 3a) refer to descriptions about what kinds of memorabilia family members collect. This often included collectibles that were purchased as a memento of a trip or event, wearable and consumable items purchased on a trip for the practical purpose of being worn or consumed, items representing personal accomplishments such as trophies or medals, and trip output items that are not deliberately purchased as a memento, but acquired in carrying out a trip (e.g., maps and pamphlets).

- Reasons for collecting/not collecting (Table 3b) refers to descriptions of why memorabilia are collected. Example reasons include to serve as a souvenir from a trip or event, or to act as a reminder of one's heritage. If participants did not collect memorabilia, this category includes descriptions of why they were not collected, such as to avoid clutter from storing them.

- Storage locations (Table 3c) refer to the physical location where memorabilia are kept. Descriptions typically included high-level areas of the home (i.e. rooms), and lower level containers or places that memorabilia are stored (e.g., on shelves or hung on walls).

- Reasons for locations (Table 3d) refer to descriptions of participant's rationale for keeping memorabilia in a particular location. This typically included reasons such as placing the item where it could be seen on display or act as a conversation piece, or placing items where there is adequate space to store them and the items do not cause a cluttered appearance.

- Associated memories (Table 3e) refer to participants' descriptions of the memories that each memorabilia item evoked. Descriptions typically included direct experiences where the item evoked a memory of a specific place or event, or indirect experiences, such as to serve as a reminder of one's heritage or as a reminder of the person that gave them the item (if it was a gift). 
Table 3: Sample analysis codes for souvenirs.

\begin{tabular}{|c|c|c|}
\hline a) Memorabilia Types & Example Codes & Description \\
\hline \multirow[t]{3}{*}{ Collectibles } & [Statues] & Statues (small or large). \\
\hline & [Dishes] & Ornamental dishes, e.g. plates, vases. \\
\hline & [Pins] & $\begin{array}{l}\text { Small pins with a picture or emblem } \\
\text { printed on them. }\end{array}$ \\
\hline \multirow[t]{2}{*}{ Wearables and Consumables } & [Food] & Food items, e.g. tea, chocolate, candies. \\
\hline & [Clothes] & $\begin{array}{l}\text { Clothing items that can be worn, e.g., } \\
\text { hats, shirts. }\end{array}$ \\
\hline Personal Accomplishment & [Trophies] & $\begin{array}{l}\text { Trophies or medals won for personal or } \\
\text { team accomplishment. }\end{array}$ \\
\hline \multirow[t]{2}{*}{ Trip Output } & [Maps] & Maps of trip locations. \\
\hline & [Pamphlet] & $\begin{array}{l}\text { Pamphlets describing a location, e.g., } \\
\text { schedule, itinerary, handouts from tours. }\end{array}$ \\
\hline b) Reasons for Collecting/Not & Example Codes & Description \\
\hline \multirow[t]{2}{*}{ Collecting } & [Roots] & $\begin{array}{l}\text { A reminder of one's heritage and cultural } \\
\text { roots. }\end{array}$ \\
\hline & [Memory] & A memory of the event or location. \\
\hline Not Collecting & [Clutter] & $\begin{array}{l}\text { Souvenirs are seen as being messy and } \\
\text { producing clutter. }\end{array}$ \\
\hline c) Storage Locations & Example Codes & Description \\
\hline \multirow[t]{3}{*}{ High-level } & [Bedroom] & A family member's bedroom \\
\hline & [Living Room] & The family's living room \\
\hline & [Office] & $\begin{array}{l}\text { Room designated as a home office, e.g., } \\
\text { where the computer is kept. }\end{array}$ \\
\hline \multirow[t]{3}{*}{ Low-level } & [Shelf] & $\begin{array}{l}\text { A shelf hanging on a wall, or case of } \\
\text { shelves }\end{array}$ \\
\hline & [Wall] & Hanging on a wall directly \\
\hline & [Storage] & A storage shelf or area in the home. \\
\hline d) Reasons for Locations & Example Codes & Description \\
\hline \multirow[t]{4}{*}{ Rationale } & [Display] & $\begin{array}{l}\text { An area that is easily visible publicly in the } \\
\text { home. }\end{array}$ \\
\hline & [Not Clutter] & $\begin{array}{l}\text { An area that won't make the items look } \\
\text { like clutter. }\end{array}$ \\
\hline & [Space] & $\begin{array}{l}\text { An area that has enough space to store } \\
\text { items. }\end{array}$ \\
\hline & [Conversation] & $\begin{array}{l}\text { An area that it can act as a conversation } \\
\text { piece. }\end{array}$ \\
\hline e) Associated Memories & Example Codes & Description \\
\hline \multirow[t]{2}{*}{ Direct Experience } & [Event] & Memories of the event. \\
\hline & [Location] & Memories of the location the item is from. \\
\hline \multirow[t]{2}{*}{ Indirect Experience } & [Roots] & Reminder of one's heritage or roots. \\
\hline & [People] & $\begin{array}{l}\text { The people who gave the item to a family } \\
\text { member. }\end{array}$ \\
\hline
\end{tabular}

We now focus our discussion around the four types of souvenirs that we saw: collectibles (individual and group), wearables and consumables, personal accomplishment, and trip output (Table 3a). Here we interweave findings reflecting the other themes identified by our codes to illustrate the locations, memories, and reasoning associated with souvenir collection and displaying routines.

\subsection{Collectibles}

Collectibles are memorabilia that represent places or events. As a group, collectibles contain by far the greatest variety in types of items. Examples include things such as decorative or artistic souvenirs from a place, statues, rocks, or postcards. Collectibles can be further broken down into individual or group collectibles. 
Individual collectibles are typically one-off items chosen to represent a specific place or event. These items can range from knick-knacks (e.g., the birds and ship in Figure 2) to those that are decorative or artistic. Collectibles are often kept on display. Figure 3, for example, shows several collectibles in one family's home. From left to right, the ornamental trees on the shelf, the painting on the wall, and the statue on the stand were all collected from various

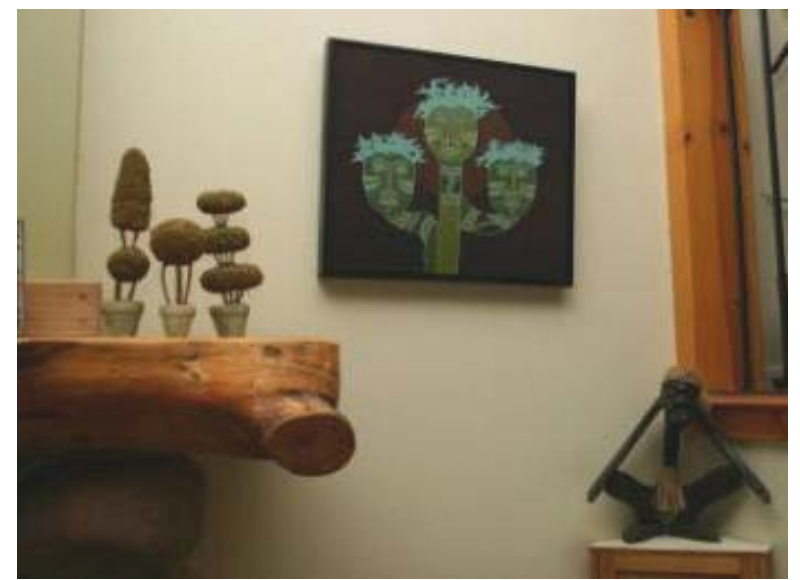

Figure 3: Example collectibles on display in the home

locations that the family has traveled. Another participant describes several of the individual collectibles on display in her home:

It's a bouquet of tulips. That would represent our trip to Holland because Holland is known for their tulips. And the reef shark there represents our trip to Fiji because we saw a lot of reef sharks...the didgeridoo, you look at it and you automatically know it is from Australia... at least I do. - P11, Wife

Some families profess to eschew memorabilia. Yet closer inspection reveals a different story. Consider our 'Banica' family participants (mother, father, and teenage daughter). They moved to Canada from Romania five years ago, and their grandmother has been staying with them on an extended visit for over a year. At the beginning of our interview, the family claimed not to have very many mementos on display. As described by the mother:

If you ask me, I don't like too many things displaying around the house. I find it tiring. I like an open space. That's why I don't like too many decorations

However, they did describe the importance of sharing photos from Romania in order to share their heritage with their new Canadian friends:

We are Canadians now, but we came from Romania five years ago and lots of friends and Canadian people wanted to see our background. So we bring some of these from Romania - to show to the church, to the people we meet, to the friends.

As the interview progressed and we were shown around various locations in the home, we found that mother, daughter, and grandmother collected a large amount of memorabilia from Romania. These items, such as art work, ornamental and religious items, etc., were placed on display and spread throughout the home - including a guest room/office, on walls in the main living area, and even in a finished basement. Figure 4 shows just a small sample of the collectibles found in the Banica family's home. As we came across these items, we found many of them led to storytelling, which gave a very detailed picture of their heritage.

This is a very well known Romanian writer, so when I see this picture I remember my background - I've been a teacher in Romania - my background, the writers, all that. 

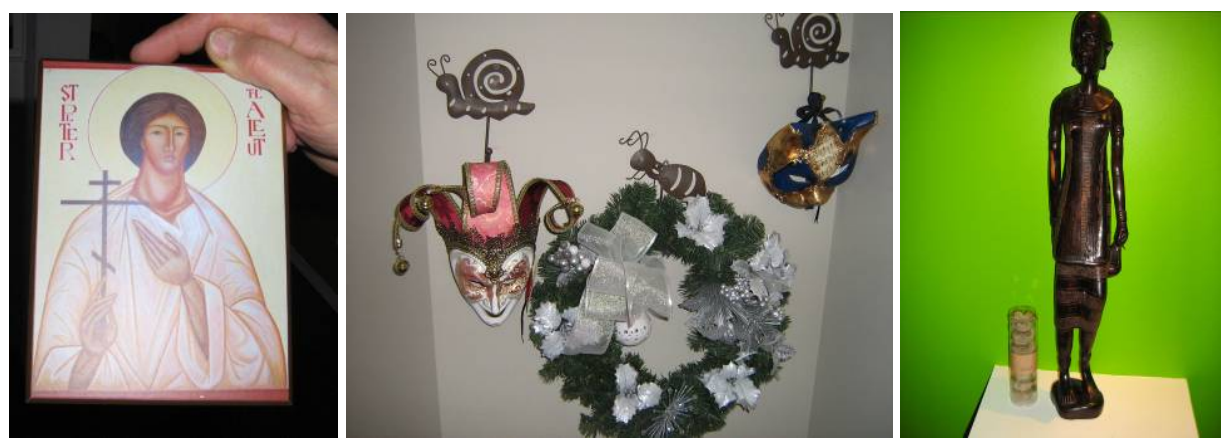

Figure 4: Examples of collectibles reflecting cultural heritage.

This I brought from Romania, we like this kind of art. When we see them we think about the places in the mountains. They have villagers...people from the village that don't have studies to do art. They do crafting...that kind of art. And I think about those places, as we used to go to the mountains. They were displaying them on the streets there.

This is very famous in Romania, it's what they call the miracle icon. The people go there...they go and prey and they get their wish there.

The family didn't have many opportunities to return home so these items, which were highly representative of their heritage, were very important in allowing them to pass cultural knowledge from parents/grandparents to their child. As the mother describes, these items allowed them to remember:

The places, the people, and feelings as they relate to our background and history and memories.

While the family may not consider these items too often in their daily life, the various items displayed in their home provide opportunities for storytelling and reflection, which was seen as an enjoyable experience.

[The tour] made me think about when you run around too much, we don't think about the stuff that we have...it was nice because in the beginning I was thinking 'Oh, what can I show?'

As with print photos, we found that the locations families chose for individual collectibles was largely motivated by pragmatic reasons, such as where there was adequate room to display them, or by aesthetics, such as where they fit in with the décor of the house. In particular, they tended to be decorative and often found on public display in various locations throughout the house, e.g., hung on walls, in bathrooms, or on shelves and mantles.

As a group, individual collectibles appear to be immediately promising for linking to photos. They are usually selected as they convey an image reminiscent of the place or event they represent. These items tend to be placed on display, often in areas for entertaining guests. Because these items are often strongly representative of the places they are from, they can become conversation pieces that in turn could lead into serendipitous opportunities for photo sharing.

Yet these opportunities are tempered by several factors. The first problem is mobility. Some items are small and robust, so they could easily be moved around or used in conjunction with a photo sharing system. For example, the religious artwork in Figure 4 (left) is highly mobile and unlikely to break. However, other items are heavy and fragile, which makes their use problematic. For example, the dishes shown in Figure 5 (top) 
(which evoke cultural memories for another family in our study) are fragile: family members would not want to move or touch them for fear of breaking them. The second problem is location. Items like the dishes in Figure 5 (top) could be in a hard to reach location - the shelf is approximately 6 feet from the floor. The third problem is that sometimes there is no option to move items at all. For example, the cloth art in Figure 5 (bottom) is fixed to its location on the wall. A fourth problem is location. While individual collectibles would most commonly be displayed in areas for entertaining guests, they could also be located in other private or less convenient places around the house. If a desired item were not nearby, it would have to be retrieved for use with a photo sharing system.

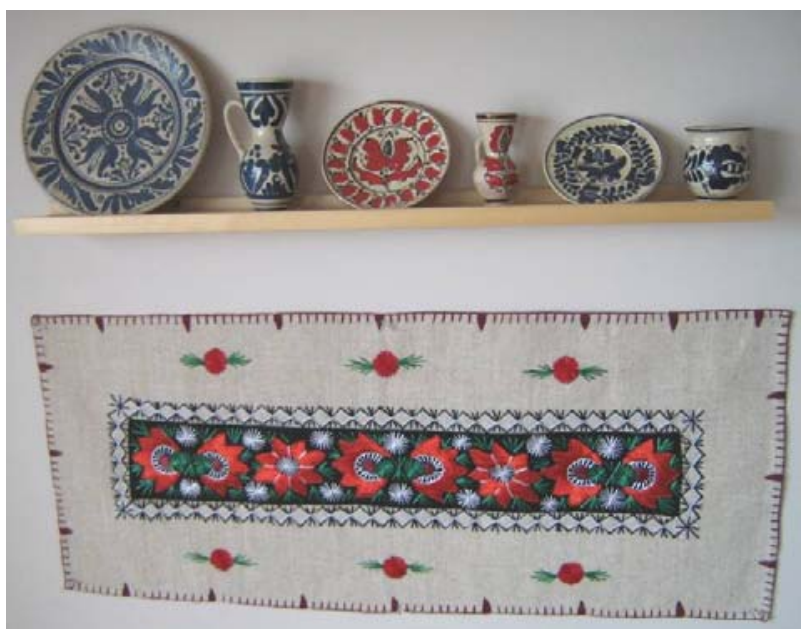

Figure 5: Fragile or difficult to move collectibles

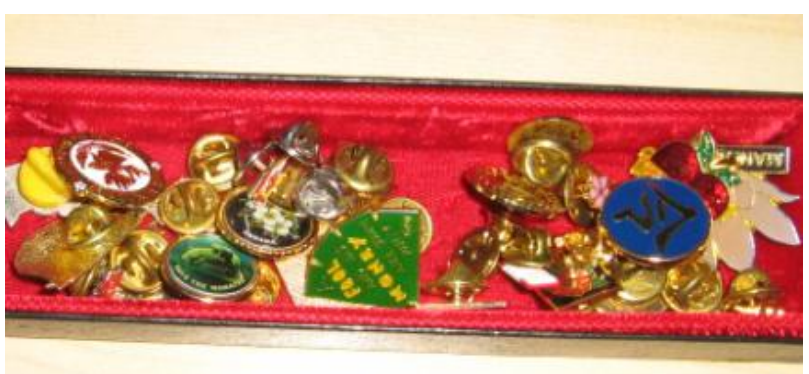

Figure 6: Group collectibles: pins kept in a drawer

Group collectibles are another sub-category of collectables, where the owner has a special interest in a certain type of object and seeks to form a collection of them. Some of the items we saw were from trips the collector had been on personally, while others were gifts from friends who knew that the person had an interest in that type of item. Examples include money, stamps, postcards and even souvenir pins from various countries (Figure $6)$.

My husband especially likes to collect stuff. He wants to do a map and have a coin from every country. - P3, Mother

As another example, consider the "James" participant family. The James' had moved to Calgary in the last few years and had begun taking family outings to go on hikes. On these hikes they took many photos, some of which were put into a framed collage and placed on display in an upstairs hallway. The mother describes her enjoyment of the display:

We did lots of hiking and I was so excited about everything we saw...and it's easy, every time I come out I pass [the photos] and I remember.

When we asked the James family about their souvenir collections they said they didn't often like to buy souvenirs. They had, however, made a regular practice of collecting stones from their various hiking trips. The stone collection had been turned into decorative pieces - they had been painted and given a label underneath with the hike they were from. The collection had then been placed on display on the living room fireplace 
ledge (Figure 7), and as the father noted it "helped give them initiative to continue [hiking]".

Group collectibles tend to consist of many small items, and as such they are often kept together in an out-ofthe-way storage such as a drawer. For example,

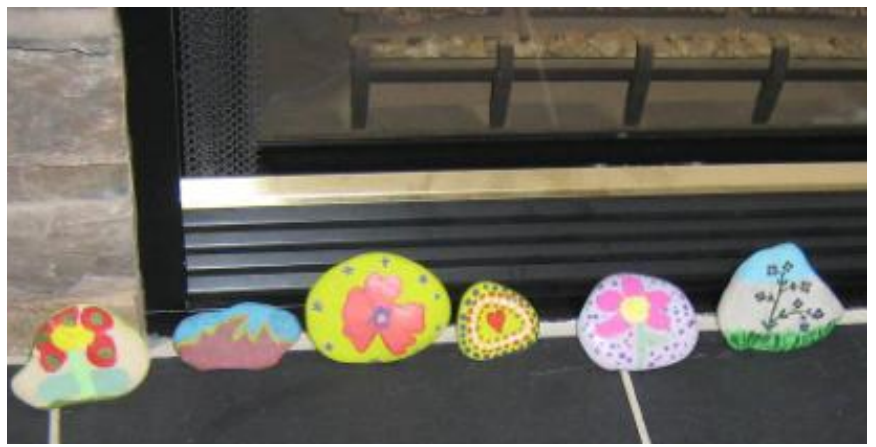

Figure 7: Group collectibles: painted rocks gathered from hikes Figure 6 shows how one family located their souvenir pin collection in a special desk drawer. Because of their likely location, certain group collectibles may not be amenable for linking to photo collections. Yet some of the more decorative group collectibles, such as the James' stones, are placed together in a public display. As these items are small and tend to be kept together, they could potentially be kept in a collectible box in a convenient place that would allow them to be used as part of a photo viewing system.

\subsection{Worn/Consumed}

Our next class of mementos, worn or consumed, includes items such as clothing, jewelry, or food that were acquired on trips and are representative of or unique to the place they are from. For example, one father told us how he would routinely bring back chocolate from his travels, as he found chocolate differed between regions or countries. Like collectibles, these items were representative of the place they were from; however, they were purchased for a more practical reason - to be worn or consumed. As such, these items would be unsuitable for linking with photos as part of a photo sharing system, so we do not discuss it further.

\subsection{Personal Accomplishment}

Personal accomplishment mementos are items that commemorate personal achievements in activities such as sports or musical performance. Items in this class typically include trophies, medals, or certificates. These items were commonly displayed; e.g., placed on shelves or framed on walls. In some instances they are displayed in public areas of the home, such as the living room. However, because they are personal, they are also often kept displayed in a personal space, typically a bedroom. For example, Figure 8 shows a display of basketball awards kept on a shelf in a teenager's bedroom.

Personal accomplishment may seem like an obvious fit for linking to photos. However, some of these mementos were too general to link to a specific set of photos. For

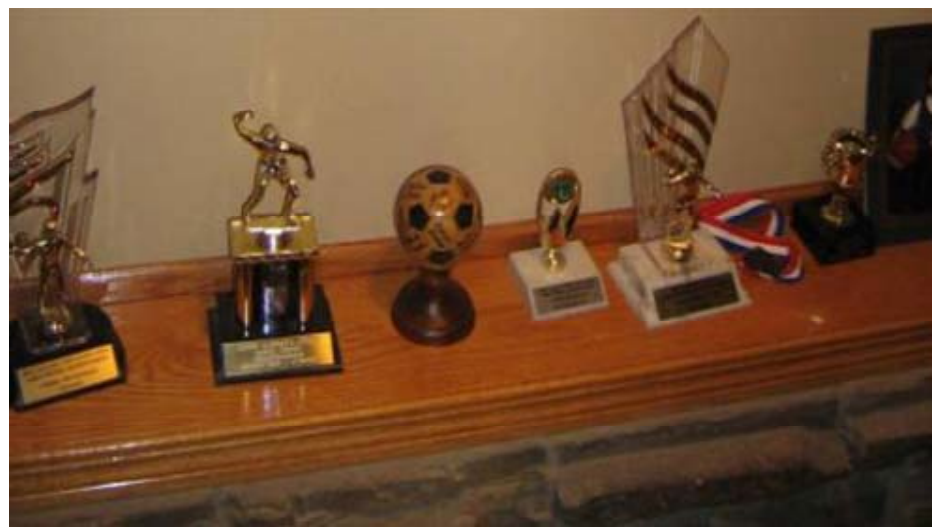

Figure 8: Trophy \& medal mementos of personal accomplishment - 20 - 
example, a trophy won at the end of a basketball season might be associated with several photo sets, e.g. photos from the season, photos from the winning game, or photos of the team. This confusion could cause difficulties for creating and remembering associations for certain items used with the system. These items also suffer from location problems, similar to individual collectibles, particularly when kept in personal spaces (e.g. bedroom).

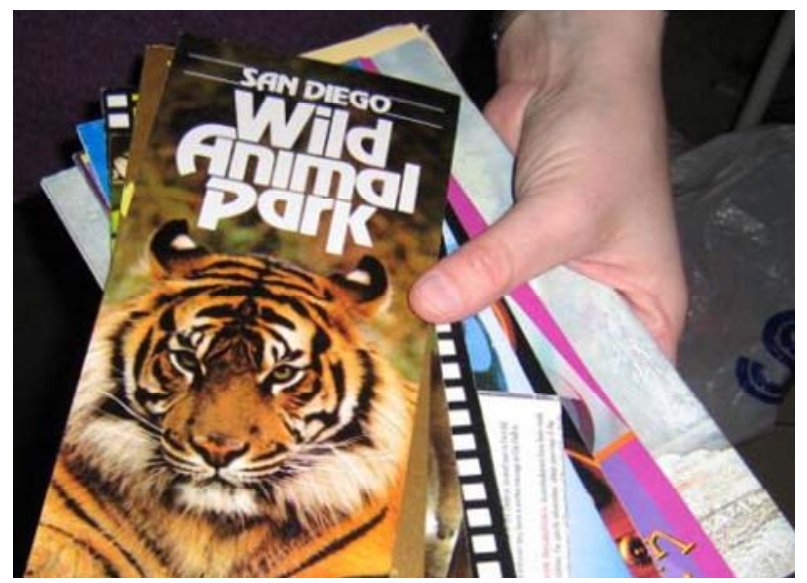

Figure 9: Maps \& brochures as trip output

\subsection{Trip Output}

The final class of mementos, trip output, is comprised of items that are gathered as a result of a trip, but unlike collectibles are not deliberately purchased as a souvenir. Instead, they are accumulated as a result of planning and carrying out the trip. Typical trip output includes items such as tickets, maps, or pamphlets (Figure 9).

While trip output items are not immediately thought of as souvenirs, nor are they considered decorative itmes. Indeed, most had no immediate practical purpose after the trip, so they were often stored away and seldom brought out. However, they proved highly valued for the memories associated with them and were rarely thrown away. That is, many families kept trip output collections to recall memories of the trips they were from.

I think I collect everything. I keep ticket stubs, receipts, brochures, and sugar packages...Originally it was because of the scrapbooks because I knew I'd have a way to save them all. And I guess it just invokes more memories of the vacation...sometimes they're funny or interesting - P7, Mother

Consider the example of the "Smith" participant family. The husband and wife went on many trips, as one was a teacher and the other volunteered and taught after-school childrens' programs. The Smiths had visited many places throughout Canada, the U.S., and worldwide. They wanted to avoid accumulating too much clutter around the house, and so they did not routinely buy souvenirs from their numerous trips. Rather, they preferred to take numerous pictures on their trips and stated that they did not have many physical souvenirs to show. However, our tour through their home revealed that they kept many trip output items, such as guidebooks, tickets, itineraries, and maps. Because these items were smaller, they could easily be kept together, sometimes placed in out-of-theway storage to avoid clutter. Figure 9 is an example of some of Smith's items, which were kept in a storage box in the basement. The wife describes another of their trip output collections:

They're just things that I can't throw away - this is from when I was in Japan - I don't want to throw them away, but I never look at them. 
Table 4: Summary of souvenir amenability for linking with photos.

\begin{tabular}{|l|l|}
\multicolumn{1}{l|}{ Souvenir Class } & Amenable For Linking \\
\hline Individual Collectibles & Yes. However, mobility/location throughout the home may be problematic. \\
\hline Group Collectibles & $\begin{array}{l}\text { Possibly. Currently kept in storage together, but could conveniently be kept for use with } \\
\text { system. }\end{array}$ \\
\hline Worn/Consumed & No. Practical use makes linking difficult. \\
\hline $\begin{array}{l}\text { Personal } \\
\text { Accomplishment }\end{array}$ & Similar to Individual Collectibles, but may be too general for linking a single photo set. \\
\hline Trip Output & Similar to Group Collectibles. Possibilities for creative re-purposing. \\
\hline
\end{tabular}

Trip output items often had associated photos, and the Smiths noted one of the advantages of keeping print photo albums was that some of the smaller related trip output items could be kept in with the albums:

I like the ones that are actually in the album, because I can put all the other little stuff I keep - like tickets, postcards, or pamphlets - I can put right in with the pictures.

The husband in the Smith family particularly liked collecting maps from his trips, as they allowed him to visualize and recall the places he had been:

I always keep maps from everywhere I go. The rest of the stuff...I kind of keep tickets and booklets. But I just like seeing the physical area I was in with the maps.

Trip output items provided a natural link to related digital photo sets as they were highly representative and documented aspects of the various trips. While they were currently kept in out of the way storage, groups of them could potentially be easily moved and kept in a convenient place for linking with photo collections.

"I'd have to figure out some way to store my maps. A lot of times when I'm on trips - with band trips we do a lot of bus travel - I literally follow where we are on the map, and maybe get to the point of highlighting the routes we're taking and making references of where we go."

\subsection{Summary and Discussion}

Table 4 gives a summary and recap of our souvenir classification. Individual collectibles seem to provide the best fit for linking with photo collections, as they are highly representative of trips or events that often have associated photos (Table 4, Row 1). As well, they are commonly placed on display in the home, allowing them to become conversation pieces that could lead to opportunities for photo sharing. However, their location throughout the home could lead to difficulties in moving them to a display for use with a photo sharing system. As typically used, grouped items, such as group collectibles (Table 4, Row 2) and trip output (Table 4, Row 5), were not immediately amenable for linking, given families' current routines. These were often kept out of the way in storage. Yet families may store such items out of the way simply because they have no practical and immediate purpose. However, the culture of how group collectibles and trip output are stored may change if systems that link physical memorabilia to photo sets become available; we will discuss this further in Section 7. Worn/consumed (Table 4, Row 3) and personal accomplishment (Table 4, Row 4) items seem the least amenable for linking physical memorabilia to photo collections.

\section{SOUVENIRS}

We now move from our study of domestic routines surrounding photos and memorabilia to one SOUVENIRS: a system that links memorabilia to photo collections as a way to promote collocated photo-sharing in the home. This section describes our first version of 
SOUVENIRS, created before the study was done. Section 7 describes how we revised SOUVENIRS based on our study results (Sections 3 \& 4), and participant reaction to it (Section 6). Of course, there are likely many ways in which one could imagine linking souvenirs with photos, and SOUVENIRS is just one example in this design space. Other examples are described in the following related work section. A video illustrating SOUVENIRS in action is also available for viewing (Greenberg and Nunes, 2008).

\subsection{Related Literature}

The idea of using tagged physical objects as handles to electronic information is well known in the tangible and ubiquitous computing fields. In particular, Photo Browser (van den Hoven and Eggen, 2003) is a hand-held device used as part of an in-home environment for memory recollection. The device provides an interface for browsing photo collections, as well as a means to send individual photos to alternate displays (e.g. a digital photo frame or TV). Photo Browser can also recognize physical souvenirs. Users can drag individual photos to be associated with a physical object, or browse the set of associated photos. Yet Photo Browser was not explicitly designed for to encourage photo-sharing: the focus of their research was for memory recollection.

Memodules (Mugellini et al., 2007) is a framework for prototyping tangible interfaces by allowing links between RFID-tagged physical objects and actions taken in the digital world. For example, they describe how a colleague's business card can trigger actions such as opening up an email composer to that colleague, and they also mention linking souvenir to photo sets. However, their focus was on presenting their technical framework, while ours was on the detailed study and design rationale of how people might use such a domestic technology to encourage photo-sharing.

Frohlich and Fennell (2007) looked at how physical memorabilia is used to retain memories in the home, and presented two systems that support storytelling around these objects. The memory shelf links an object to a recorded audio message. The anniversary plinth prints out a textual record of the objects history. They do not draw the link between photos and memorabilia. Similarly, Petrelli, Whittaker and Brockmeier (2008) review and describe several methods for designing digital mementos that "bridge the divide between physical and digital memories”.

Various researchers have also considered collocated storytelling over digital images through specialized devices. We know this happens regularly: people often use the preview mode of their digital cameras and cell phones to show particular photos to others. Special handheld devices have been researched and prototyped for this purpose, e.g., Balabanovic et al. (2000). Many others are investigating special software for photo sharing over digital tables, e.g., Shen et al., (2002). All these systems emphasis storytelling within a photoset, while SOUVENIRS is centered on bringing particular photosets into action. Thus the related work is complementary to our own.

\subsection{Scenario: Souvenirs in Action}

The scenario described below and illustrated in Figure 10 highlights the primary features of the first version of our SouvENIRS system, as shown to our study participants. We do not delve deeply into its technical details, as such systems are not that difficult to build. The only unusual feature is our hardware; we used Phidgets to implement our RFID technology and to build our custom scroll wheel (Greenberg and Fitchett, 2001), and we programmed the Wii controller using Brian Peek's WiimoteLib package (http://www.codeplex.com/WiimoteLib).

On a recent hiking trip to Alaska with his family, Bob took many photos with his digital camera. Bob has also brought back an interesting-looking rock he found during his 


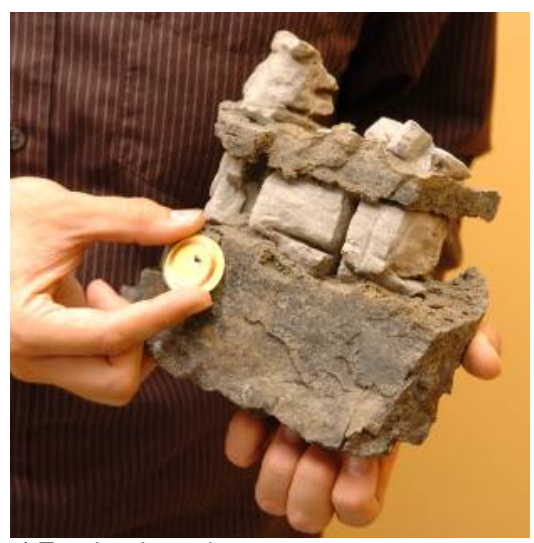

a) Tagging the rock

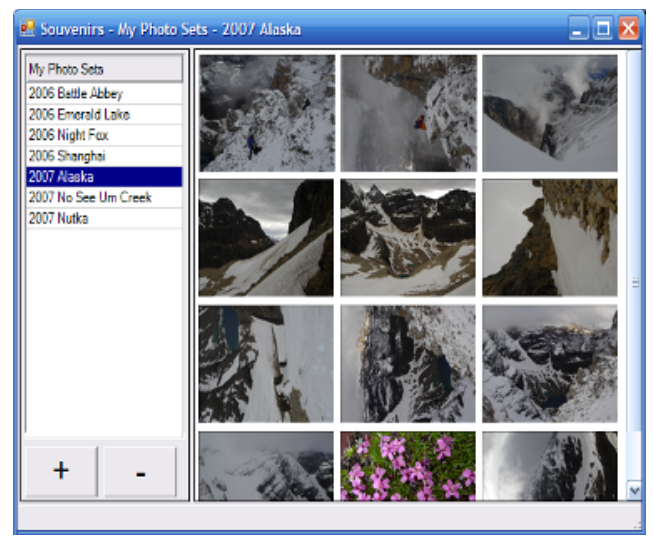

b) Adding the Alaska photos

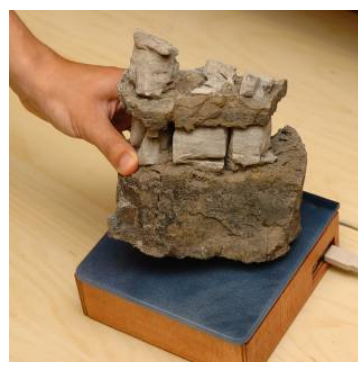

c) linking the rock to photos

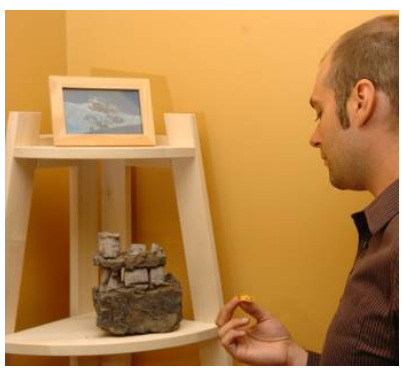

d) displaying the rock

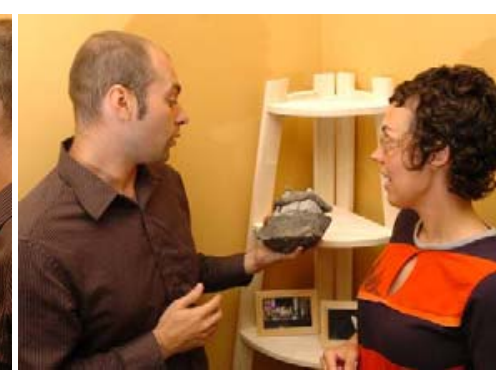

e) Conversing over the rock

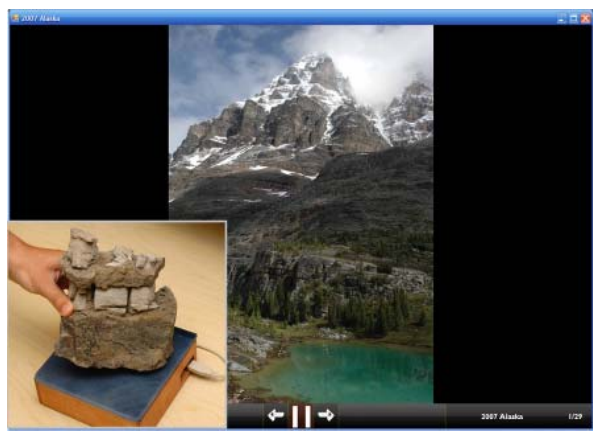

f) Starting the slide show from the rock

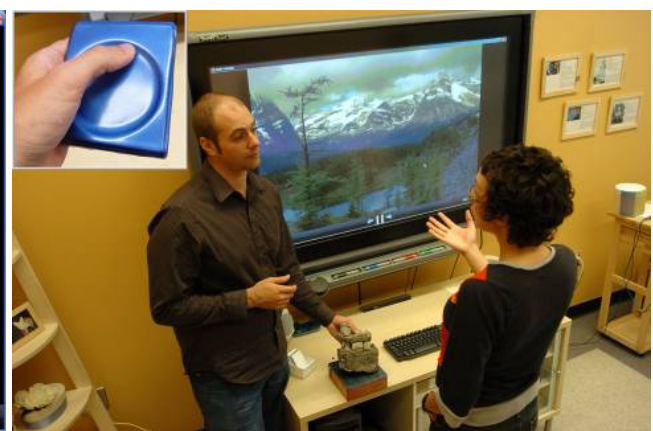

g) Storytelling through the slide show

Figure 10: SOUVENIRS in action, showing linking a memento and a photo set, and the social use of the memento to activate a slide show

hike, something he does on most his trips. After Bob returned home, he downloaded his photos into a folder on his home media center computer that is attached to a large plasma television display in his living room. He also places the rock on a display shelf in his living room, alongside other memorabilia collected from other trips.

Bob decides to use this rock as a link to his Alaskan hiking pictures. He achieves this through the following quick steps, performed in under a minute.

1. He places a sticker - a small pre-glued RFID tag - on the bottom of the rock (Figure 10a).

2. He starts SouvenIRS so it is displaying on his television screen (Figure 10b). He drags the folder containing his Alaska pictures onto the SouvEnIRS window. SoUvENIRS imports these photos, Bob renames the photo set to '2007 Alaska'. Alternately, Bob 
could have mixed and matched photos from several folders by dragging and dropping them into the SouvenIRS window. This photo set is now available to be linked.

3. He places the rock on a platform (an enclosed RFID reader) located next to the TV. It reads the RFID sticker identity, and associates that sticker (and thus the rock) with that photo set. (Figure 10c)

4. He returns the rock to the display shelf (Figure 10d).

Somewhat later, Bob's family returns home and are having tea in the living room. His wife, Alice, sees the rock sitting on the mantle, and mentions it. Bob picks up the rock, reminding her that it is his Alaska memento, and asks if she wants to see the Alaska photos (Figure 10e). She assents, and he places the rock on the platform (Figure 10f); the photo set immediately plays as a slide show (Figure 10g). Using a specially designed physical scroll wheel (Figure 10g inset), or a Nintendo Wii controller, Bob and Alice can cycle through photos at various speeds and/or pause them (not shown).

Two months later, Alice has a book club event in her home. Coincidentally, the book being discussed is John Krakauer's Into the Wild, a non-fictional and ultimately tragic account of a young man who went off to live in the Alaska outback. Some of the book club members had never been to Alaska, and Alice asks if they want to see what the terrain looks like. While she has no idea where Bob has actually stored the photos, she easily remembers that the rock is linked to them. She shows the rock to the group and mentions that Bob collected it when he was up there. As she is discussing details of that trip, she places the rock on the RFID platform; the slide show begins as before. After the show (and several glasses of wine), the book club members talk about the other memorabilia on the display shelf, and Alice story-tells about some of the places the family has travelled to. She selectively shows a few photos from each place by passing particular memorabilia items over the platform.

\subsection{Design Rationale}

In Bob's family, linking the rock to the Alaska photo set makes the rock a symbolic link to the photo set. Because the rock is then displayed in the home, it becomes a social instrument - an autotopography where the arrangement of these objects constitute " $a$ physical map of memory, history and belief” (Petrelli, Whittaker and Brockmeier, 2008). He and his family can take advantage of the rock's meaning, location and visibility, without being concerned of how photos are stored and how they can be accessed on disk. The result is serendipitous photo viewing and storytelling.

More generally, the design of SOUVENIRS assumes that physical memorabilia can become a handle to access particular digital photo sets, just as a URL serves as a handle to rich sets of digital information. As personal artefacts, these memorabilia have physical presence, are positioned in the home in a meaningful way to its inhabitants, and can trigger recollection and story-telling in their own right (Section 4; also see Petrelli, Whittaker and Brockmeier, 2008). Thus the linkage of photos is a natural extension of how people associate memories with the artefact, which then works as a natural lead-in to the photo show through opportunistic story-telling.

Of course, paper-based framed photos and photo albums serve similar purposes, as they too are memorabilia. The power of SOUVENIRS is that it works without requiring the home occupant to print and organize their photos, an operation that is increasingly expensive given that people take and store many more digital vs. film photos. In its stead, a single physical artefact serves as an access point for a photo set.

In Section 3.6, we summarized several differences between digital and print photos, where these differences inhibit collocated digital photo sharing. Here, we describe our design rationale for mitigating these problems. 
Locations are exploited. Digital photos are located on computers that are hidden, hard to access, and not necessarily in locations amenable to sharing. By using memorabilia as a handle to these photos, the advantages and meanings of memorabilia location serves as a surrogate to photo location.

Serendipity re-occurs. Digital photos are not readily visible in the home to spark spontaneous sharing. The public location of memorabilia, particularly of displayed collectibles in public rooms, reintroduces opportunity for serendipity.

Accessibility by circumventing access control. We previously described how, in many homes, the primary digital photo organizer often stores photos on a personal passwordprotected account on a home computer. While a person may not intend to limit access to their photo set, other family members may be reluctant (or do not have permission) to log onto another person's account. SOUVENIRS is constructed in a way where digital photos are automatically published in a public file store; no access control is required.

Accessiblity by circumventing navigation problems. People often do not know about photo collections created by the primary organizer. Even if they do, they may not know how to find them, as they may be hidden in a hierarchy, have cryptic names, or stored in a database by a software system that they are unfamiliar with. Using memorabilia as a handle to photos circumvents these navigational problems, as the associated photoset is immediately displayed.

Accessibility by removing time delays. With standard digital photos, people have to stand around and wait as: the photo shower turns on and logs onto a computer; navigates through a traditional GUI system to find the photo set; starts the software and navigate through its controls to display the photos as a slide show. With SouvenIRS, one simply brings the memento to the display and the photos are retrieved immediately.

Viewing is in a social setting. Groups no longer have to view photos by crowding around small desktop computer displays located in out of the way corners of a home such as in a den or bedroom office. We assume that SOUVENIRS is permanently linked to a large display - a new generation television set such as a 50" plasma display - located in a public home space such as a living room. Unlike home offices or spare bedrooms, these rooms and the display are already set up as a social space - a space that often contains family memorabilia. Family and visitors regularly inhabit that space (which creates social opportunities), and the display is easily and comfortably viewable by all (e.g., televisions are normally surrounded by couches).

\section{SOUVENIRS AS A TECHNOLOGY PROBE}

We showed participants of our study a video of the SouvENIRS system described above. We wanted to elicit their reactions to SOUVENIRS, and gathered suggestion of how to improve its design. As we will see, this technology probe re-affirmed some of our design rationale, but also suggested places where SOUVENIRS could be improved.

\subsection{Sharing and Tangible Objects}

Participants had positive reactions towards SouvenIRS' affordances for digital photo sharing within the home, and the use of memorabilia as tangible objects for retrieving photo sets. They liked how the system was situated in the home's social context, i.e., the use of the large television display in a public home area to show the photos, and how this setting created an opportunity for multiple onlookers to view and discuss the photos. As one participant put it: 
That's something I'd show friends. More friends can watch at the same time, not like an album where only a maximum of two people can look at an album. I think it's good. - P1, Mom

Additionally, people liked how SouvenIRS hid the technology and the tedium of retrieving and sharing photos as compared to traditional computer navigation. They liked how it was immediately "ready to go" at any time, and how it avoided the need to navigate and invoke applications to show digital photos. Some saw this as particularly useful for showing pictures to elder relatives who could have trouble with conventional computers.

Where it would be useful is for the parents and grandparents. If they could just do that to their TV. Never mind the issue of having to transfer all of the pictures over. Once it was setup then my parents could just wave an object. - P7, Husband

If you found an object to link it to or a picture it would be really easy to find an album. And because it is already connected to the TV or a big screen it is easier than connecting the camera. - P9, Fiancée

People were generally receptive to the idea of using tangible objects as a link to retrieving photo sets. They said this would allow sharing "by chance" (serendipity) from displayed memorabilia. They liked the novelty of physical linking, and thought it was something they would want to show off to friends. They thought that linking photos to physical objects would be easy and would make finding / displaying photo sets quicker and more enjoyable than with traditional computers.

It seems a lot more fun and interesting to have a symbol from the actual place rather than having to go on your computer and start clicking on folders. They are a lot more organized this way and there'd be more memories. - P3, Teenage Daughter

You don't have to sit down and try to find the picture you want to show friends, it's just there. - P1, Dad

\subsection{Challenges}

Participants also noted several concerns about the SouvenIRS design. These are important, as they suggest redesign directions.

Fit to existing practices. Families stated that the utility of physical memorabilia as a link to photos depended on the actual practices they used to display souvenirs and mementos in the home. Some noted that they preferred not to have many mementos displayed around the home for aesthetic reasons, and thus questioned the overall usefulness of physical objects as a links. (Although recall that several families who thought they did not have many mementos actually did have quite a few). In those cases, they still thought the large public display was very desirable.

I would like it if all of the pictures that I had on the computer were on the TV and I could scroll them, have a remote, and look through them. That would be great. Linking them to objects would be trouble for me because I don't like to keep those kinds of objects...that part of it wouldn't work as well for me. - P13, Husband

Scalability was a related concern. People who did keep mementos were worried that religiously linking photos to physical mementos kept in accessible places would eventually lead to clutter and storage problems. They stated they took and maintained large collections of photos, which would exacerbate this problem. 
You would have to remember to get something each time...after a while you'd need a large storage area beside your TV...I'd have to store a lot. - P1, Father

The amount of pictures that we take, we'd have boxes of items, you'd be literally grabbing things from boxes. For us I think a big screen like that if you could hook it up to this [remote]. The biggest problem we have is crowding when we show photos, but to be able to put that stuff on to a bigger screen would be good. - P16, Husband

However, people suggested a solution to this problem. As well as physical mementos, they wanted the option of having an "index box" containing single physical photos or descriptive cards, each tagged and thus linking to its associated digital photoset. The box would allow many tagged photos or cards to be kept together without clutter. Others noted that an on-screen photo list might be just as effective.

I'd have a hard time finding and storing those objects. Where would I put them all? One picture for each set would work. - P2, Mother

The object thing is the thing that I'd find hard because you want to have the objects close to your TV...I'd almost rather have folders where you could just touch, but we're not really souvenir-type people...I guess a box of cards would work, but if you're just going to do that you may as well have folders on the screen. - P7, Wife

Appropriately associating physical items to photos was another concern. Some thought they might have difficulty finding a memorable item that appropriately links to a given photo set. In some cases, there simply may not be any relevant item available.

The only thing is it applies more to pictures from something special like maybe a party or wedding where you could have something that triggers the pictures. But with just regular family pictures it would be hard and sometimes you do take a lot of those pictures, and sometimes you do want to retrieve some of those pictures. - P8, Mother

For the Turkey trip, we could link that [points to an item brought back], but for family photos it would be trickier to find something. - P9, Fiancé

Some also mentioned that the association between an object and a photo set may not be known to other members of the family, and so it would be difficult for others to tell that an object was actually linked to a set of relevant photos. People also wondered if they would forget the association between a given memorabilia and its photos. Some even suggested writing descriptions on the physical items.

Over time you might forget what souvenir was attached to what group of pictures, especially if you went somewhere twice. Like if you went to Seattle twice and took two sets of pictures. It's a good way to remember but also not organized enough for me. I'd maybe just have a normal object, like a stick with a sensor, then you could write the date, time, and event name and then just put that over the sensor. - P14, Daughter

One family was initially reluctant about the idea of SouvENIRS for their own personal use, as they thought it would be more useful for families that routinely bought souvenirs and mementos. However, once they realized they could use the system with their trip output items, they caught onto the idea of repurposing them for use with the system.

You could put tags on all the different places on your maps. 
That would be cool. Yeah. Or pages in a guidebook actually, that would be really neat.

Breaking and losing mementos. Participants were concerned that moving physical mementos around to use as links to photo sets would increase the risk that fragile items could break, or expensive items lost. If this happened, they were also concerned that those photo sets could then become inaccessible.

So what happens if you lose the object that has the tag on it? Do you lose the photos? - P1, Father

\section{DISCUSSION AND SYSTEM REVISION}

Many of our findings supported the original SouvENIRS design. However, they also introduced concerns. Here, we describe how several of these concerns, along with our own thoughts of other possible problems, resulted in design changes in the next SOUVENIRS iteration. We also describe how social practices may change as systems such as SOUVENIRS is used in the home, and technical suggestions that make such systems more practical.

\subsection{Souvenirs Version 2.}

Creating public photo sets. While people said they liked the idea of accessing photos from their TV (as well as from other computers), this begs the question of how the photos get there. The issue is that people, by default, store photos in their own accounts rather than in a public place, and that they likely will want to share only a subset of their photos rather than all of them.

To solve this problem, we revised SOUVENIRS to work across all computers on a home network. Individual home occupants can now start the photo management view of SOUVENIRS on whatever computer and account they want. Similar to other photo management software, this view allows them to create new photo sets: as in Figure 10b, photo sets are displayed as a list, and photos are represented by thumbnails. When a family member starts SouveNIRS on another computer, that instance locates the photos on the original computer, downloads them, and caches them locally (through a configuration dialog, people can specify where SouvENIRS should look for photos). Meta-data that describes associations between photo sets and RFID tags are copied as well. Thus, all photos and meta-data are available to any other connecting SouvENIRS client. At this point, other family members can view, modify, or even remove links and photo sets as desired from whatever home computer they happen to be using.

Photo sharing and linking is optional. It is unrealistic to expect every single photo set to have an associated physical handle (Kirk et al., 2006). Somewhat similar to how people currently create photo albums around specific events or favored photos vs. organizing all photos, we now expect people to selectively choose photos to share and link, where a large bulk of photos will be left behind. In our changed version, only photos dragged into SOUVENIRS are used by SOUVENIRS. That is, unlike other photo sharing systems, SOUVENIRS does not search all hard drives

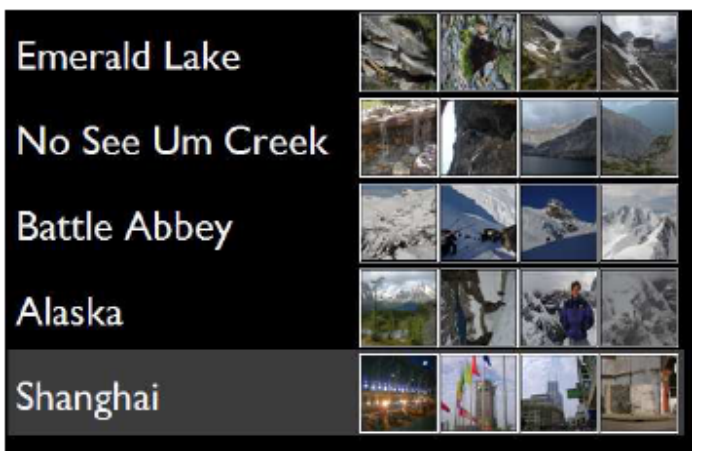

Figure 11:The revised navigation screen - 29 - 
within a home network for photos. Only those that are explicitly dropped into the system are made available across the various computers in the home.

We also changed SouvenIRS so that people, by default, could access and display all photo sets through traditional on-screen navigation. They use the modified photo management view in Figure 11, which lists photo sets and thumbnail samples. To start a slide show, people can now navigate and select a set from this list using standard input devices, or our physical scroll wheel control, or a Nintendo Wii controller. Thus, people can share a photo set without requiring a tagged object. This is useful in the case the tagged object has been broken or lost, or when people have forgotten which object is associated with a photo set, or when no associated object is available.

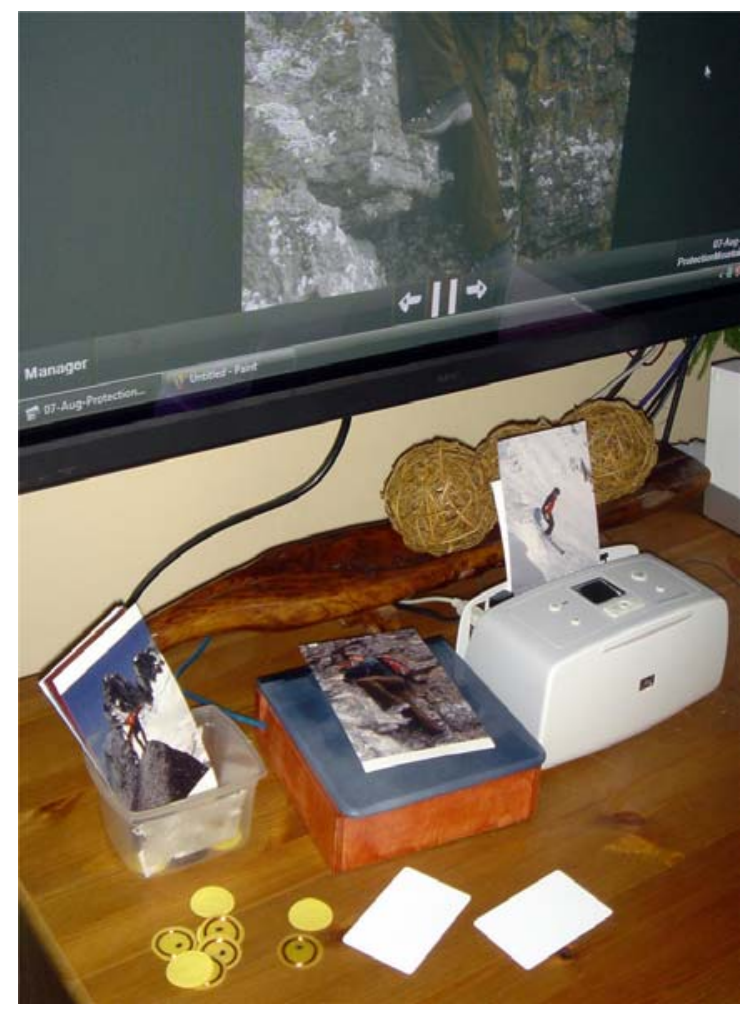

Figure 12: Photos, RFID cards, and printers

A further advantage is that these lists are usually in chronological order. Thus if a family brings up a photo set using a tagged object, photo sets - some which may be untagged - that are taken at the same time are shown on the list. This increases serendipity of a tagged object leading to serendipitous photo-sharing of multiple photo sets.

At any point, people can decide to create a physical link to a photo set simply by setting a tagged unassigned memento over the reader. In this way, people can adopt the technology to best fit their practices, using mementos as little or as much as they want.

Using other physical objects as links. People suggested that mementos were not always practical, e.g., because of size, or fragility, or because no memento is available, or because they are hard to organize. They also suggested other physical objects would be suitable, such as printed photos and index boxes.

We modified SouvENIRS in two ways to make it easy for people to create custom mementos that can be linked to digital photos, where these custom mementos are easily organized. We now package SouvENIRS with a dedicated small one-click photo printer, 5x7 photo sheets, a box of sticky-backed RFID tags, a box of credit-card sized RFID cards, and a marking pen. As shown in Figure 12, these should be located by the large television. First, a person can now quickly print an exemplar photo from the photo set being viewed and stick an RFID tag onto that print. Second, people can annotate the RFID cards with written descriptions and text. For both print photos and cards, people then quickly link them to a photo set by passing it over the RFID reader. Both print photos or cards could perhaps be stored and organized in time/event order in attractive boxes kept next to the display (Figure 12, bottom left), or in booklets dedicated to 
particular types of events, or (for photos) even within photo albums or framed on a nearby photo wall.

Using other displays, particularly mobile ones. While we envisaged SOUVENIRS as running on the public family television screen, it became apparent that this was overly restrictive. As mentioned by our participants, some mementos aren't movable, either because they are fixed in place, or are too large or heavy to move, or are too fragile, or are too far from the public screen. Clearly, people need to be able to view photo sets associated with such objects spread around the home. As well, there may be opportunities presented by computers in other home locations. While these obviously include personal computers, we are especially interested in mobile displays such as laptops and tablet PCs, as these can be moved around the home.

As mentioned, we modified SOUVENIRS so that it works over a home network. Consequently, people can now access photos from any computer on that network. As a SOUVENIRS client connects to the server, any new photo sets since the last connection are cached on the local computer to make navigation and display responsive.

Next, we created a mobile photo-frame by attaching a small RFID reader onto a tablet PC. Although this is technically identical to the large display configuration, this mobility leads to a different social practice of photo sharing akin to how people show snapshots while sitting around a table. As shown in Figure 13 (top left), a family member has just retrieved photos attached to a displayed collectible (a small statude) via the mobile photoframe. She shows it and manually passes it around people sitting around her. Thus, SOUVENIRS no longer demands that the physical object be moved to the large screen.

Pick \& Drop. The small screen size and limited viewing angle of most mobile devices means that they are comfortably viewed by perhaps two or three people. For larger groups and for easier viewing, we still believe that photos are best seen on the large display. To let people move from small devices to large displays, we designed the mobile picture frame to act as a go-between the tagged artifact and the large display using a 'pick and drop' strategy (Rekimoto, 1997). We already saw how the mobile picture frame raises the photo set associated with a tagged object (Figure 13, top left). If this group decides to move to the large television, the person simply moves the picture frame to the platform by the large television (Figure 13, top right), and the photo set will then appear on that display for all to see as before (Figure 13, bottom). Technically, we embed an RFID tag on each mobile device. When a photo set is selected on the mobile device, the device's tag is temporarily

associated with the chosen photo set. In this way, the device can be passed over an

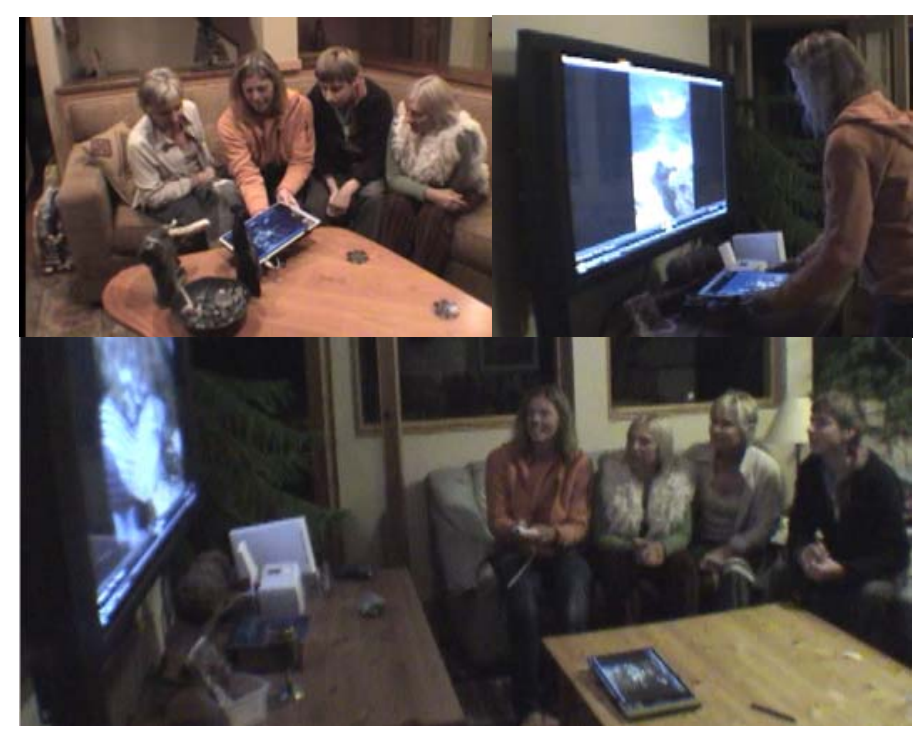

Figure 13.SOUVENIRS, the mobile photo-frame, and pick \& drop - 31 - 
RFID base attached to the large display and the photo set will be triggered.

Remembering and knowing associations. Another concern was that people said they may not know or remember what a physical item is linked to. While we argue that shared knowledge would develop as items are displayed and families adopt the system, we do have alternate solutions that encourage discovery of particular photo-sets attached to memorabilia. In particular, a person can use the mobile photo-frame to serendipitously "examine" what photos are attached to mementos as one moves around the home. Indeed, we envisage games where children in the family could play a form of photo "hide-andseek" or "hot/cold", where they have to find particular photos attached to objects around the home. Hiding photos engages the parents, while finding them engages the children.

However, the issue remains that these objects do not directly show that they are being used as links - particularly as they might co-exist with other displayed items that are not being used in this way. One other option is to have geo-located RFID tags, where the mobile computer could show a photo index of all tags in its field of view. However, such technology is currently prohibitively expensive.

\subsection{Evolving Creative and Social Practices}

Thus far we have discussed design revisions to our SouvENIRS prototype with the intent of providing a better fit with the existing practices seen in our study interviews.

Here, we consider how the introduction of a SOUVENIRS style system could change a family's cultural and social practices over time. While we have yet to observe how the system is adopted through long-term installations in family homes, the interviews and our own self-trials also allow us to speculate on how families might develop new practices given the availability of a system such as SOUVENIRS - particularly in encouraging creative use of tagged objects.

Family practices are not static; we expect they will change (hopefully positively) to create meaning around the technical and social artifacts we introduce. If adopted, a system like SOUVENIRS could increase a family's desire to collect memorabilia specifically for use as links to photos. As well, a family's practice of displaying memorabilia could change, where they specifically locate memorabilia with the benefit of keeping them near the public display. Similarly, a family might put greater consideration into how their homes are arranged to naturally allow storytelling through displayed souvenirs and photos.

What is tagged could also change. We hypothesized that displayed collectables are the most amenable memento for photo-linking, but other memorabilia types could be exploited. For example, trip output items (e.g. guidebooks, maps, etc.) are typically stored away as they have no practical purpose after the trip. Yet, they are strongly linked to memories, and often have related photo sets. The opportunities of photo-linking may change family storage practices of these items. In our own experience setting up SOUVENIRS in one of the researcher's home, the family tagged and moved some of their travel guide-books (previously stored in a cupboard) to a shelf near the television. This shelf of guidebooks became the mementos linking to photo sets of the major trips they had been on over the years. Indeed, multiple tags were placed on one of the guidebooks, where each corner represented a different subset of photos taken on that trip. Other ideas include tagging a wall map.

Similarly, people may reorganize their photographs to match particular memorabilia types. For example, while we argued that personable accomplishment mementos are unlikely candidates for linking, a family may (for example) decide to select highlights from photos taken over a season to portray their child's successes. That is, the 
memorabilia may introduce new meanings into how people structure their photo collections.

Changes in technology can spark further creative ideas. For example, if representative photos could be printed with a barcode, they could be immediately used as links (assuming bar code reader technology). These photos could then be organized as an album, which in turn serves as an index to various digital photo sets. Or the family could intermix photos and group collectibles in a scrap-book (as is often done), where a barcode label could be affixed near the particular group collectible item.

Another possibility is to associate physical objects with a time period rather than a particular photo set. Instead of jumping directly into a slide show, the system could show the set of photos taken around that time period (e.g., similar to Figure 11). Objects could include diary pages or calendars, each with pre-printed bar codes.

While these ideas may be speculative, they illustrate the creative potential of linking physical memorabilia to digital photos, and how they could change the practice of collocated photo viewing.

\subsection{Technical suggestions}

The design revisions previously discussed expand on the features present in our software prototype, and aim to provide a better fit with the practices observed in the study. From a design perspective, these features would (hopefully) increase the likelihood that such a system for linking photos and memorabilia could be adopted in real homes. However, even with these revisions, our own experience with the revised prototype of SOUVENIRS illustrated that the deployment of the system in an actual home was still difficult. Through our own self-trials, we noted several issues that need to be resolved in order to make a system for linking digital photos to physical memorabilia practically deployable in real homes.

First, the linking functionality must be integrated within a robust and well-liked software package for managing and viewing digital photos, such as Google's Picasa (picasa.google.com). As the focus of our research is to investigate linking digital photos to physical memorabilia, rather than on building a photo management/viewing system, we had hoped to incorporate this functionality by tapping into an existing photo management API, but none were available. As such, we implemented the SoUVENIRS photo management/viewing functionality from scratch. While usable, it is not as robust or complete as a commercial system. Additionally, integration with a popular photo management system would allow its users to adopt it without having to re-organize their photos with another system.

Second, a protocol for easily discovering, sharing and maintaining photo sets over the home network is needed. Our prototype implementation makes use of a shared folder as a central store, where photos are both gathered centrally from other household computers, and disseminated as a cache to all connecting clients in the interest of providing interactive speed. While workable, it does require setting several configuration options. This setup may be difficult for the average home user, and can be inefficient in terms of hard disk space required as the shared photo collection grows. Because of these factors, a more robust solution to sharing photos over a home network is needed.

Next, limitations of hardware items in the revised prototype were problematic. Taping an RFID tag-reader to the back of our tablet PC worked, but was not perfectly reliable due to its proximity with interfering signals generated by the PC. As well, the RFID reader platform needs to be tuned to detect items within a certain distance. If the range is too close, it will not detect tags that are not attached to the bottom of a physical object. If the range is too long, it will detect tags attached to nearby physical objects that may have been moved off the platform. 
We also anticipate an alternate and simpler device for pick-and-drop. While the tablet PC allowed pick and drop, it is too cumbersome to be used more generally. Another solution is a small RFID device with a pen form factor. Using the pen, one can "pick" several tagged memorabilia located around the home, then "drop" them onto the home display.

Finally, systems like SOUVENIRS should extend beyond slide-show photo showing by integrating it with domestic photo display technologies. Researchers are now considering how traditional print photos displayed in the home create meaning for its inhabitants, and looking at how systems for digital photo display (such as in digital photo frames) could behave similarly (Kim and Zimmerman, 2006; Swan and Taylor, 2008). We know that framed prints and physical mementos are often intermingled within domestic displays. Perhaps placing a digital photo frame amongst mementos would automatically let one select and display a photo related to those mementos.

\section{CONCLUSION}

We have investigated how physical memorabilia can be used as opportunities to move into home-based collocated digital photo sharing. This work covered three major stages: investigating family photo and memorabilia routines through contextual interviews, a design exploration by building a system that links physical objects and photos, and design reflection through a form of technology probe. Our findings show that there is indeed promise for using memorabilia to encourage collocated photo sharing. We believe that systems such as SOUVENIRS could eventually be used by families to overcome the current limitations of digital photo sharing in the home, and that new social practices will evolve over time.

We recognize that SouvENIRS represents only one possible design direction. We encourage other researchers to build on our findings and explore other design possibilities for utilizing physical artifacts found throughout the home as links to abstract digital collections.

\section{ACKNOWLEDGMENTS}

This research was partially supported by NSERC through its Scholarship, Discovery Grant, and NECTAR Network Grant programs.

\section{REFERENCES}

BALABANOVIC, M., CHU.L, AND WOLFF, G. 2000. Storytelling with Digital Photographs. Proc ACM CHI'oo, 564-571.

BEYER, H. and HOLTZBLATT, K. 1998. Contextual Design: Defining Customer-Centered Systems. Morgan Kaufmann.

CHALFEN, R. 1987. Snapshot Versions of Life. Bowling Green, Ohio: Bowling Green State University, Popular Press.

CRABTREE, A., RODDEN, T., AND MARIANI, J. 2004. Collaborating around Collections: Informing the Continued Development of Photoware, Proc ACM CHI'04.

CSIKSZENTMIHALYI, M. and ROCHBERG-HALTON, E. 1981. The Meaning of Things: Domestic Symbols and the Self, Cambridge University Press.

DOURISH, P. 2001. Where the Action Is: The Foundation of Embodied Interaction. MIT Press.

FROHLICH, D., AND FENNELL, J. 2007. Sound, Paper and Memorabilia: Resources for a Simpler Digital Photography. Personal Ubiquitous Comput. 11 (2), 107-116.

FROHLICH, D., KUCHINSKY, A., PERING, C., DON, A., ARISS, S. 2002. Requirements for Photoware. PrOC ACM CSCW'02.

GREENBERG, S., AND FITCHETT, C. 2001. Phidgets: Easy Development of Physical Interfaces through Physical Widgets. Proc ACM UIST '01, 209-218 
GREENBERG, S. and NUNES, M. (2008) Sharing Digital Photographs in the Home by Tagging Memorabilia (Video). Research report Report 2008-917-30, Department of Computer Science, University of Calgary, Calgary, Alberta, Canada.

HOLTZBLATT, K., WENDELL, J., AND WOOD, S. 2005. Rapid Contextual Design: A How-To Guide to Key Techniques for User-Centered Design, Morgan Kaufmann.

HUTCHINSON, H., MACKAY, W., WESTERLUND, B., BEDERSON, B., DRUIN, A., PLAISANT, C., BEAUDOUIN-LAFON,M., CONVERSY, S., EVANS, H., HANSEN, H., ROUSSEL, N., and EIDERBACK, B. (2003). Technology probes: inspiring design for and with families. In Proc ACM CHI’03, 17-24.

KIM, J., AND ZIMMERMAN, J. 2006. Cherish: smart digital photo frames for sharing social narratives at home. CHI '06: CHI '06 extended abstracts on Human factors in computing systems, 953-958.

KIRK, D., SELLEN, A., ROTHER, C., AND WOOD, K. 2006. Understanding Photowork. Proc ACM CHI '06, 761-770.

LINDLEY, S. and MONK, A. (2006). Designing appropriate affordances for electronic photo sharing media. In CHI '06: CHI '06 extended abstracts on Human factors in computing systems, pages 1031-1036, New York, NY, USA. ACM Press.

MILLER, A., AND EDWARDS, W.K. 2007. Give and Take: A Study of Consumer Photo-Sharing Culture and Practice. Proc ACM CHI '07, 347-356.

MUGELLINI, E., RUBEGNI, E., GERARDI, S., AND KHALED, O. 2007. Using Personal Objects as Tangible Interfaces for Memory Recollection and Sharing, Proc TEI '09 $1^{\text {st }}$ Intl Conf Tangible and Embedded Interaction, Baton Rouge, USA.

NEUSTAEDTER, C., ELLIOT, K., AND GREENBERG, S. 2006. Interpersonal Awareness in the Domestic Realm. Proc. OZCHI, 2006.

NUNES, M. (2008) Sharing Digital Photographis in the Home Through Physical Memorabilia. Master's thesis, Department of Computer Science, University of Calgary, Calgary, Alberta, Canada, September.

NUNES, M., GREENBERG, S. and NEUSTAEDTER, C. (2008) Sharing Digital Photographs in the Home through Physical Mementos, Souvenirs, and Keepsakes. In Proceedings of the ACM Conference on Designing Interactive Systems - ACM DIS'08. (Cape Town, South Africa), ACM Press, pages 250-260, February 25-27.

NORMAN, D. 2003. Emotional Design: Why We Love (or Hate) Everyday Things. Basic Books.

PETRELLI, D., WHITTAKER, S., and BROCKMEIER, J. 2008. AutoTopography: what can physical mementos tell us about digital memories? Proc. ACM CHI'08, 53-62.

REKIMOTO, J. 1997. Pick-and Drop: A Direct Manipulation Technique for Multiple Computer Environments. Proc ACM UIST '97. 31-39.

RODDEN, K., AND WOOD, K. 2003. How do people manage their digital photographs? Proc ACM CHI '03, 409-416.

SHEN, C., LESH, N., VERNIER, F., FORLINES, C., AND FROST, J. 2002. Sharing and Building Digital Group Histories. Proc ACM CSCW '02, 276-290.

STRAUSS, A., AND CORBIN, J. 1998. Basics of Qualitative Research: Techniques and Procedures for Developing Grounded Theory, SAGE Publications.

SWAN, L., AND TAYLOR, A.S. 2008. Photo displays in the home. DIS2008: Proceedings of the $7^{\text {th }}$ ACM Conference on Designing Interactive Systems, 261-270.

TAYLOR, A., SWAN, L., AND DURRANT, A. 2007. Designing Family Photo Displays, Proceedings of the Tenth European Conference on Computer-Supported Cooperative Work, 24-28, September 2007, Springer.

VAN DEN HOVEN, E., and EGGEN, B. 2003. Digital photo browsing with souvenirs. Proceedings of Interact 2003, IOS Press, 1000-1003.

VAN DEN HOVEN, E., and EGGEN, B. 2005. Personal souvenirs as ambient intelligent objects. Proceedings of the 2005 Joint Conference on Smart Objects and Ambient Intelligence, New York, NY, USA. ACM Press, 123-128. 